CRYSTALLOGRAPHIC COMMUNICATIONS

ISSN 2056-9890

\title{
Crystal structures of the gold NHC complex bis(4-bromo-1,3-diethylimidazol-2-ylidene)gold(I) iodide and its 1:1 adduct with trans-bis(4-bromo- 1,3-diethyl-imidazol-2-ylidene)diiodidogold(III) iodide
}

\author{
Rolf Büssing, ${ }^{a}$ Ingo Ott ${ }^{\mathrm{a}}$ and Peter G. Jones ${ }^{\mathrm{b}}$ *
}

anstitut für Medizinische und Pharmazeutische Chemie, Technische Universität Braunschweig, Beethovenstr. 55, D38106 Braunschweig, Germany, and ${ }^{\mathbf{b}}$ Institut für Anorganische und Analytische Chemie, Technische Universität Braunschweig, Hagenring 30, D-38106 Braunschweig, Germany. *Correspondence e-mail: p.jones@tu-bs.de

Edited by C. Schulzke, Universität Greifswald, Germany

Keywords: crystal structure; gold; halogen bonds; $\mathrm{N}$-heterocyclic carbene.

CCDC references: $2119423 ; 2119422$

Supporting information: this article has supporting information at journals.iucr.org/e

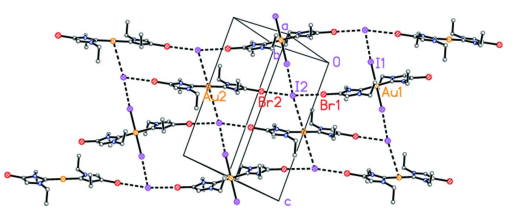

\section{Chemical context}

Gold complexes have been used in medicine since ancient times and have been applied as drugs for the treatment of rheumatoid arthritis since the 1930s. Currently, gold species are being actively investigated in inorganic medicinal chemistry as possible anticancer agents or anti-infectives (Mora et $a l ., 2019)$. Some of the existing therapeutics have reached the clinical trial stage as a result of drug repurposing efforts. Metal $\mathrm{N}$-heterocyclic carbene (NHC) complexes in general have also proved to be biologically and medicinally active compounds (Ott, 2020); in particular, gold complexes with NHC ligands are often synthesized and investigated because of the high stability of the gold-carbon bonds and the convenient synthetic access to a broad variety of structurally diverse NHC structures (Nahra et al., 2021). We have reported on the synthesis, characterization and biological effects of [bis(4bromo-1,3-diethyl-imidazol-2-ylidene)gold(I)] iodide (3) (Schmidt et al., 2017a) (Fig. 1). Notably, this complex and related derivatives triggered cytotoxicity against cancer cells, showed a low serum protein binding, and inhibited growth of some pathogenic bacteria. Furthermore, we have recently investigated various gold NHC complexes as antibacterial agents and inhibitors of bacterial thioredoxin reductase (Büssing et al., 2021).

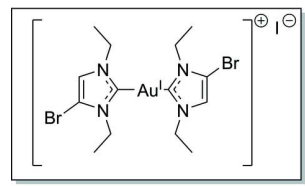

3

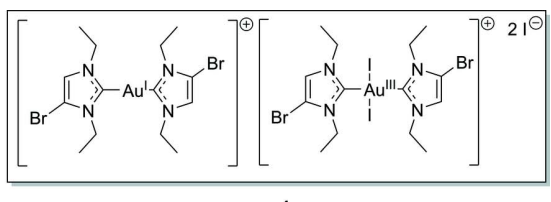

4 


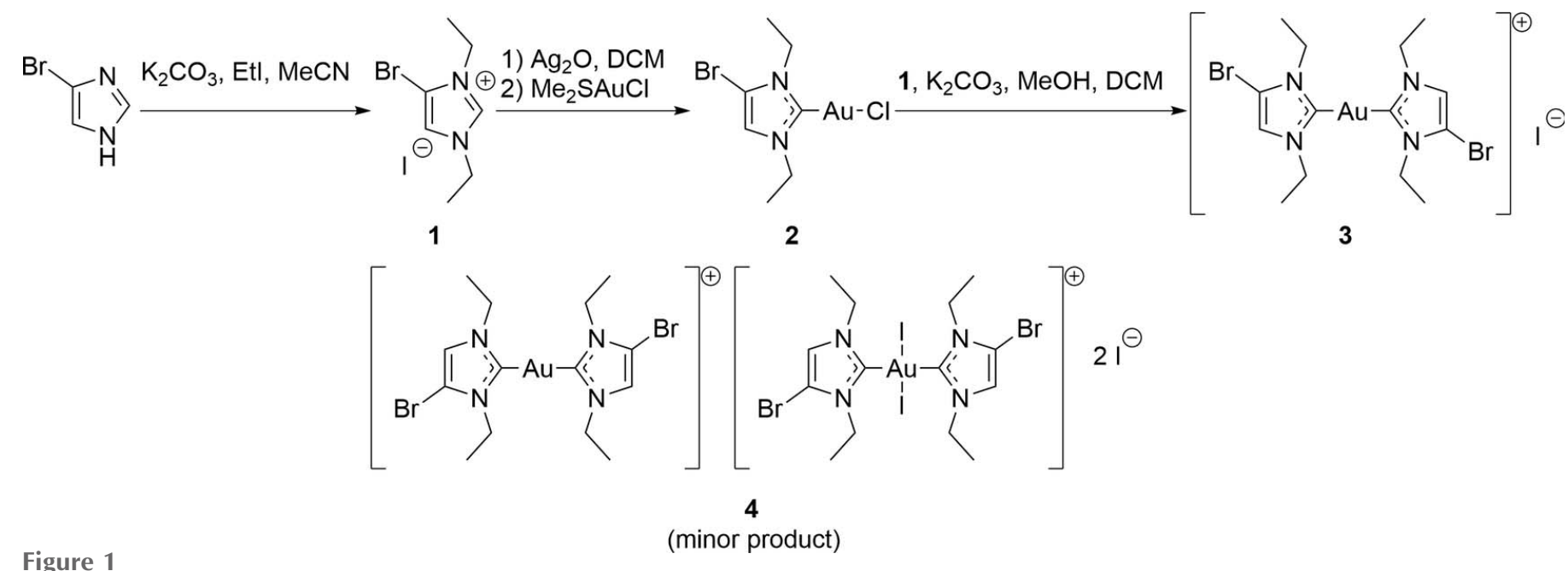

Figure 1

Synthesis of compound $\mathbf{3}$, recrystallization of which also afforded a small amount of separable crystals of compound 4 .

Here we report the structure of $\mathbf{3}$, together with that of its 1:1 complex (4) with trans-[bis(4-bromo-1,3-diethyl-imidazol2-ylidene)diiodidogold(III)] iodide, formally its $\mathrm{I}_{2}$-oxidized $\mathrm{Au}^{\mathrm{III}}$ analogue; the latter was formed in small quantities when 3 was recrystallized. Further studies on the bioinorganic and medicinal chemistry of $\mathbf{3}$ and related derivatives are the subject of ongoing projects.

\section{Structural commentary}

The structure of the asymmetric unit of $\mathbf{3}$ is shown in Fig. 2. All atoms lie on general positions in space group $P \overline{1}$. Selected intra- and intermolecular dimensions (including contact

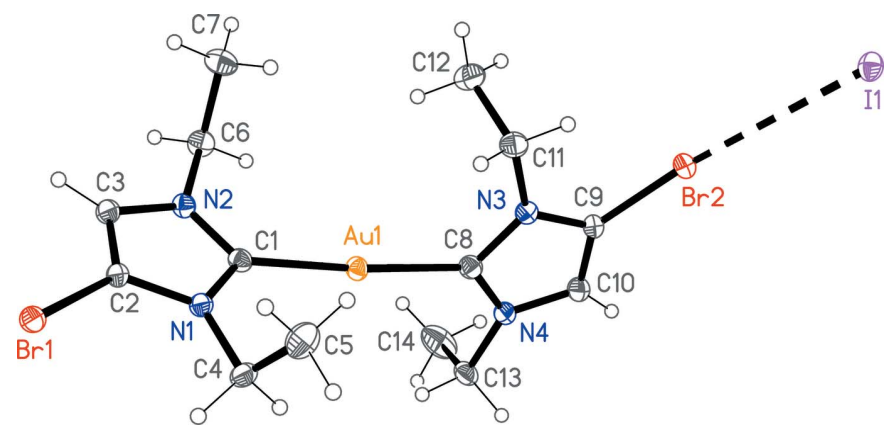

Figure 2

Structure of the asymmetric unit of compound $\mathbf{3}$; ellipsoids represent $50 \%$ probability levels. The dashed line indicates a halogen bond.

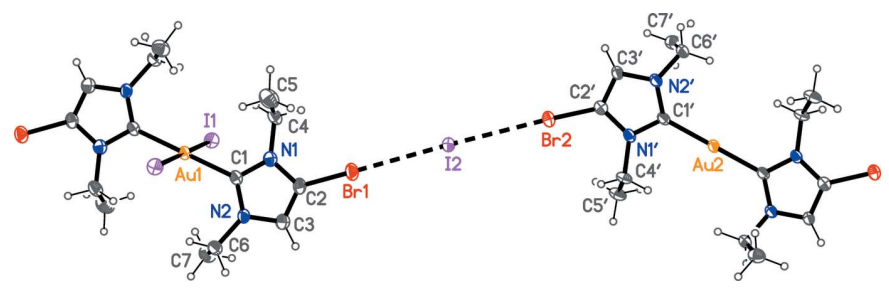

Figure 3

Structure of compound 4; the asymmetric unit has been extended by symmetry to show complete cations. Ellipsoids represent $50 \%$ probability levels. The dashed lines indicate halogen bonds. distances) are presented in Table 1. The gold atom is, as expected, linearly coordinated. The NHC planes subtend an

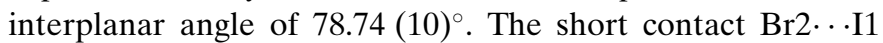
seen in Fig. 2 is one of two such contacts that determine the crystal packing (see next section).

The structure of compound $\mathbf{4}$ is shown in Fig. 3. Selected metrical parameters for intra- and intermolecular interactions (including contact distances) are presented in Table 2. Both gold atoms lie on inversion centres; the $\mathrm{C}-\mathrm{Au}-\mathrm{C}$ and $\mathrm{I}-$ $\mathrm{Au}-\mathrm{I}$ angles are thus exactly linear, and the NHC planes of both cations are exactly coplanar. The gold(III) centre displays the expected square planar geometry. The $\mathrm{Au}-\mathrm{C}$ bond is slightly longer than in 3. For further discussion, see Database survey below.

Table 1

Selected geometric parameters $\left(\AA,^{\circ}\right)$ for $\mathbf{3}$.

\begin{tabular}{lcll}
\hline $\mathrm{Au} 1-\mathrm{C} 1$ & $2.020(2)$ & $\mathrm{I} 1 \cdots \mathrm{Br} 2$ & $3.6072(3)$ \\
$\mathrm{Au} 1-\mathrm{C} 8$ & $2.022(2)$ & $\mathrm{Au} 1 \cdots \mathrm{Br} 2$ & $3.8033(3)$ \\
$\mathrm{I} 1 \cdots \mathrm{Br} 1^{\mathrm{i}}$ & $3.5294(3)$ & & \\
& & & \\
$\mathrm{C} 1-\mathrm{Au} 1-\mathrm{C} 8$ & $174.97(9)$ & $\mathrm{C} 2-\mathrm{Br} 1 \cdots \mathrm{I} 1^{\mathrm{iii}}$ & $172.43(7)$ \\
$\mathrm{Br} 1^{\mathrm{i}} \cdots \mathrm{I} 1 \cdots \mathrm{Br} 2$ & $101.436(8)$ & $\mathrm{C} 9-\mathrm{Br} 2 \cdots \mathrm{I} 1$ & $162.21(8)$ \\
\hline Symmetry codes: & (i) $x-1, y-1, z+1 ;$ & (ii) $-x+1,-y+1,-z+1 ; \quad$ (iii) \\
$x+1, y+1, z-1$. & & &
\end{tabular}

Table 2

Selected geometric parameters $\left(\AA,^{\circ}\right)$ for $\mathbf{4}$.

\begin{tabular}{lclc}
\hline $\mathrm{Au} 1-\mathrm{C} 1$ & $2.033(7)$ & $\mathrm{Au} 2-\mathrm{Cl}^{\prime}$ & $2.018(7)$ \\
$\mathrm{Au} 1-\mathrm{I} 1$ & $2.6564(5)$ & $\mathrm{I} 2 \cdots \mathrm{Br} 2$ & $3.5575(8)$ \\
$\mathrm{I} 1 \cdots \mathrm{I} 2^{\mathrm{i}}$ & $3.5136(7)$ & $\mathrm{I} 2 \cdots \mathrm{Au} 1^{\mathrm{i}}$ & $4.1539(5)$ \\
$\mathrm{Br} 1 \cdots \mathrm{I} 2$ & $3.4347(8)$ & & \\
& & & \\
$\mathrm{C} 1^{\mathrm{ii}}-\mathrm{Au} 1-\mathrm{C} 1$ & 180.0 & $\mathrm{Br} 1 \cdots \mathrm{I} 2 \cdots \mathrm{Br} 2$ & $169.87(2)$ \\
$\mathrm{C} 1^{\mathrm{ii}}-\mathrm{Au} 1-\mathrm{I} 1$ & $89.05(18)$ & $\mathrm{I}^{\mathrm{i}} \cdots \mathrm{I} 2 \cdots \mathrm{Br} 2$ & $72.852(17)$ \\
$\mathrm{C} 1-\mathrm{Au} 1-\mathrm{I} 1$ & $90.95(18)$ & $\mathrm{Br} 1 \cdots \mathrm{I} 2 \cdots \mathrm{Au} 2^{\mathrm{iv}}$ & $74.604(16)$ \\
$\mathrm{Au} 1-\mathrm{I} 1 \cdots \mathrm{I} 2^{\mathrm{i}}$ & $176.29(2)$ & $\mathrm{I} 1^{\mathrm{i}} \cdots \mathrm{I} 2 \cdots \mathrm{Au} 2^{\mathrm{iv}}$ & $168.488(16)$ \\
$\mathrm{C} 2-\mathrm{Br} 1 \cdots \mathrm{I} 2$ & $179.5(2)$ & $\mathrm{Br} 2 \cdots \mathrm{I} 2 \cdots \mathrm{Au} 2^{\mathrm{iv}}$ & $114.871(16)$ \\
$\mathrm{C} 1^{\prime}-\mathrm{Au} 2-\mathrm{C} 1^{\prime i i i}$ & 180.0 & $\mathrm{C} 2^{\prime}-\mathrm{Br} 2 \cdots \mathrm{I} 2$ & $177.0(2)$ \\
$\mathrm{Br} 1 \cdots \mathrm{I} 2 \cdots \mathrm{I} 1^{\mathrm{i}}$ & $97.240(19)$ & & \\
\hline
\end{tabular}

Symmetry codes: (i) $-x,-y,-z$; (ii) $-x-1,-y-1,-z$; (iii) $-x+2,-y+2,-z+1$; (iv) $x-1, y-1, z$ 


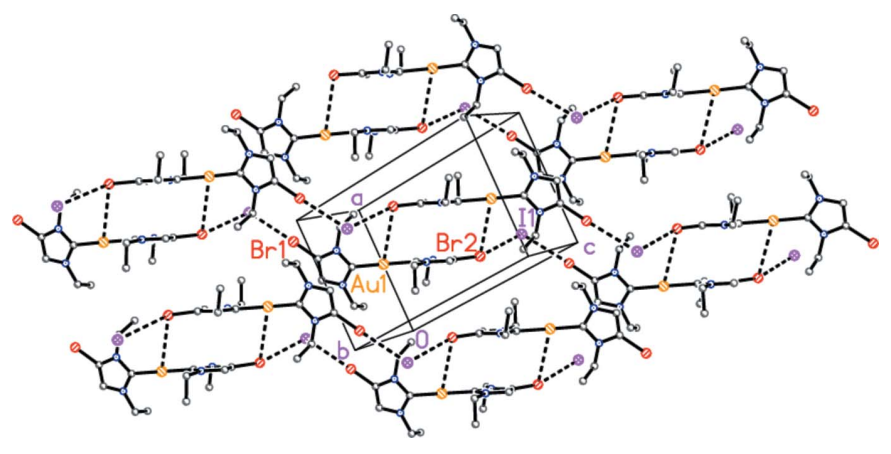

Figure 4

Packing diagram of compound $\mathbf{3}$ viewed perpendicular to (011). Hydrogen atoms are omitted. Dashed lines indicate halogen bonds or $\mathrm{Au} \cdots \mathrm{Br}$ interactions. Atom labels correspond to the asymmetric unit

\section{Supramolecular features}

The packing of compound $\mathbf{3}$ is shown in Fig. 4. It is dominated by short $\mathrm{Br} \cdots \mathrm{I}$ contacts (Table 1) that may be considered as halogen bonds (for reviews, see Metrangelo, 2008 and Cavallo et al., 2016). The $\mathrm{C}-\mathrm{Br} \cdots \mathrm{I}$ angles are approximately linear, whereas $\mathrm{Br} \cdots \mathrm{I} \cdots \mathrm{Br}$ is approximately a right angle. The anions and cations are connected to form chains with overall direction parallel to [111] . The chains are in turn connected in pairs by the contact $\mathrm{Au} \cdots \mathrm{Br} 2[3.8033$ (3) $\AA$, operator $1-x$, $1-y, 1-z]$. Within the double chains, the intercentroid distance between the carbene rings based on $\mathrm{N} 1$ and $\mathrm{N} 2$ is 3.5265 (14) $\AA$, and between the double chains the intercentroid distance between the rings based on N3 and N4 (operator $1-x, 2-y,-z$ ) is 3.6187 (14) $\AA$; these offset contacts may represent $\pi \cdots \pi$ interactions.

The packing of compound 4 (Fig. 5) also involves halogen bonds. The cations are connected to form chains parallel to [331] (horizontal in Fig. 5) by contacts between each bromine atom and the iodide $\mathrm{I} 2$. As in $\mathbf{3}$, the $\mathrm{C}-\mathrm{Br} \cdots \mathrm{I}$ angles are approximately linear. The $\mathrm{Au}^{\mathrm{III}}$ cations are further connected

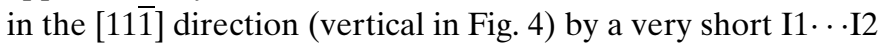
contact and a long I $2 \cdots$ Au 2 contact. The result is a reticular layer structure parallel to (1 $1 \overline{1} 0)$, in which the iodide anion I2 is four-coordinate. The angle between the two chain directions is $76.4^{\circ}$. There are no short contacts between ring centroids.

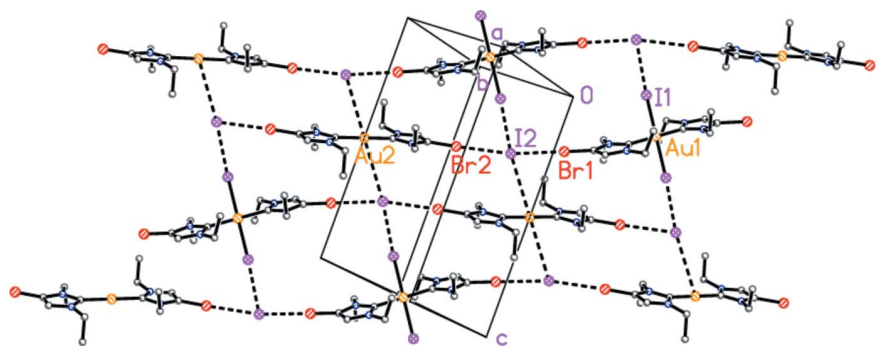

Figure 5

Packing diagram of compound 4 viewed perpendicular to (103). Hydrogen atoms are omitted. Dashed lines indicate halogen bonds or $\mathrm{Au}$... I contacts. Atom labels correspond to the asymmetric unit.
Contact distances and angles involving the heavy atoms are included in Tables 1 and 2. Some $\mathrm{C}-\mathrm{H} \cdots \mathrm{Br}$ and $\mathrm{C}-\mathrm{H} \cdots \mathrm{I}$ contacts are listed in the supporting information; these might be considered as borderline hydrogen bonds.

\section{Database survey}

Using version 2.0.5 of the CSD (Groom et al., 2016), a ConQuest search (Bruno et al., 2002) for bis(carbene)gold(I) cations gave 355 hits, with an average $\mathrm{Au}-\mathrm{C}$ bond length of $2.023 \AA$. For $\mathrm{Au}^{\mathrm{III}}$ cations of the form $\left[(\text { carbene }){ }_{2} \mathrm{Au} X_{2}\right]^{+}(X=$ halogen), only 38 hits were recorded, and only six of these involved iodine as the halogen [refcodes: ANUJIE (Baron et al., 2016), CIVMOK (Jothibasu et al., 2008), MEZZOI (GilRubio et al., 2013), POYHOB (Ghosh \& Catalano, 2009), XOMFIR and XONCAH (Holthoff et al., 2019)]. XOMFIR presents a rare example of a non-cyclic carbene ligand. The average $\mathrm{Au}-\mathrm{C}$ and $\mathrm{Au}-\mathrm{I}$ bond lengths are 2.034 and $2.614 \AA$, respectively. The $\mathrm{Au}-\mathrm{C}$ bond lengths of $\mathbf{3}$ and $\mathbf{4}$ may thus be considered normal, whereas the $\mathrm{Au}-\mathrm{I}$ bond of $\mathbf{4}$ is longer than all those previously reported. It is tempting to suggest that this is associated with the halogen bonding, but MEZZOI and POYHOB also display short I $\cdots$ I contacts ( 3.680 and $3.478 \AA$, respectively), while XONCAH has a short Au $\cdots$ I contact of $3.438 \AA$. Short halogen $\cdots$ halogen contacts between $\mathrm{Au}^{\text {III }}$ species are relatively frequent; we recently drew attention to such contacts in $\mathrm{AuCl}_{4}{ }^{-}$and $\mathrm{AuBr}_{4}{ }^{-}$salts with protonated amine cations (Döring \& Jones, 2016) but we did not include $\mathrm{AuI}_{4}{ }^{-}$salts because these are far more difficult to access.

\section{Synthesis and crystallization}

We have described the syntheses of compounds $\mathbf{1}, \mathbf{2}$ (Schmidt et al., 2017b) and $\mathbf{3}$ (Schmidt et al., 2017a) elsewhere, but give a brief summary here. The reagents were purchased from Sigma-Aldrich, Alfa Aesar or TCI and used without additional purification steps. All reactions were performed without precautions to exclude air or moisture. In the first step, 4-bromoimidazole was reacted with ethyl iodide in the presence of potassium carbonate to yield the bisalkylated imidazolium iodide (1) (Fig. 1). Compound $\mathbf{1}$ was then transformed in a two-step procedure by reaction with $\mathrm{Ag}_{2} \mathrm{O}$ and chlorido(dimethylsulfide)gold(I) to the gold(I) NHC complex 2. The biscarbene complex $\left[(\mathrm{NHC})_{2} \mathrm{Au}\right]^{+} \mathrm{I}^{-}(\mathbf{3})$ was obtained by further reaction of $\mathbf{2}$ with $\mathbf{1}$.

Single crystals of complex $\mathbf{3}$ were obtained by diffusion of $n$ hexane into a solution of $\mathbf{3}$ in chloroform/deuterochloroform. A few crystals of the mixed-valence complex $\mathbf{4}$ also formed, for reasons that are not clear, and the compound was identified by X-ray analysis as reported here.

\section{Refinement}

Crystal data, data collection and structure refinement details are summarized in Table 3. For both structures, the methyl groups were refined as idealized rigid groups allowed to rotate but not tip (AFIX 137; C-H $0.98 \AA$ A $\mathrm{H}-\mathrm{C}-\mathrm{H} 109.5^{\circ}$ ). The 
Table 3

Experimental details.

3

Crystal data

Chemical formula

$M_{\text {r }}$

Crystal system, space group

Temperature (K)

$a, b, c(\AA)$

$\alpha, \beta, \gamma\left({ }^{\circ}\right)$

$V\left(\AA^{3}\right)$

Z

Radiation type

$\mu\left(\mathrm{mm}^{-1}\right)$

Crystal size (mm)

Data collection

Diffractometer

Absorption correction

$T_{\min }, T_{\max }$

No. of measured, independent and

observed $[I>2 \sigma(I)]$ reflections

$R_{\text {int }}$

$(\sin \theta / \lambda)_{\max }\left(\AA^{-1}\right)$

Refinement

$R\left[F^{2}>2 \sigma\left(F^{2}\right)\right], w R\left(F^{2}\right), S$

No. of reflections

No. of parameters

$\mathrm{H}$-atom treatment

$\Delta \rho_{\max }, \Delta \rho_{\min }\left(\mathrm{e} \AA^{-3}\right)$

\author{
$\left[\mathrm{Au}\left(\mathrm{C}_{7} \mathrm{H}_{11} \mathrm{BrN}_{2}\right)_{2}\right] \mathrm{I}$ \\ 730.04 \\ Triclinic, $P \overline{1}$ \\ 100 \\ 8.4676 (2), 8.8248 (3), 14.0119 (5) \\ 76.374 (3), $85.320(2), 85.251$ (2) \\ 1011.99 (6) \\ 2 \\ Mo $K \alpha$ \\ 12.74 \\ $0.09 \times 0.06 \times 0.05$
}

XtaLAB Synergy, HyPix

Multi-scan (CrysAlis PRO; Rigaku OD, 2021)

$0.751,1.000$

$84327,9374,8185$

0.043

0.840

$0.023,0.052,1.02$

9374

203

H-atom parameters constrained

$1.53,-2.02$
4

$\left[\mathrm{Au}\left(\mathrm{C}_{7} \mathrm{H}_{11} \mathrm{BrN}_{2}\right)_{2}\right]\left[\mathrm{AuI}{ }_{2}\left(\mathrm{C}_{7} \mathrm{H}_{11} \mathrm{BrN}_{2}\right)_{2}\right] \mathrm{I}_{2}$ 1713.88

Triclinic, $P \overline{1}$

100

8.0245 (4), $8.5782(3), 15.9814$ (6)

91.228 (3), 96.517 (4), 92.255 (4)

1091.77 (8)

1

Mo $K \alpha$

13.23

$0.08 \times 0.03 \times 0.01$

XtaLAB Synergy, HyPix

Multi-scan (CrysAlis PRO; Rigaku OD, 2021)

$0.703,1.000$

61886, 6378, 5409

0.048

0.704

$0.039,0.105,1.06$

6378

215

$\mathrm{H}$-atom parameters constrained

$3.97,-2.66$

Computer programs: CrysAlis PRO (Rigaku OD, 2021), SHELXS (Sheldrick, 2008), SHELXL2017 (Sheldrick, 2015) and XP (Siemens, 1994).

methylene and NHC ring hydrogens were included using a riding model starting from calculated positions $(\mathrm{C}-\mathrm{H}=0.99$ or $0.95 \AA$ respectively). The $U_{\text {iso }}(\mathrm{H})$ values were fixed at 1.2 (for methylene groups) or 1.5 (for methyl groups) times the $U_{\text {eq }}$ value of the parent carbon atoms.

The asymmetric unit of $\mathbf{3}$ was chosen to include the short Br2 $\cdots$ I1 contact. This means that the iodide lies outside the reference unit cell. Similarly, the asymmetric unit of 4 was chosen as a central I2 anion coordinated by two cations (Fig. 2). The long and narrow shape of this unit means that the centroid of the $\mathrm{Au}^{\mathrm{III}}$ cation does not lie within the reference cell. In both cases, this leads to a CheckCIF Alert G.

The large difference peaks close to Au2 and 12 of structure 4 may be a consequence of its moderate crystal quality (somewhat irregular and diffuse reflection shapes) and/or residual absorption errors. The peaks can of course be made smaller by cutting the data to a lower $2 \theta_{\max }$ value, but we prefer not to do this because the mean $I / \sigma(I)$ value at highest resolution $(0.74$ $0.71 \AA$ ) is still quite high at 8.4.

\section{Acknowledgements}

We acknowledge support by the Open Access Publication Funds of the Technical University of Braunschweig.

\section{References}

Baron, M., Tubaro, C., Basato, M., Isse, A. A., Gennaro, A., Cavallo, L., Graiff, C., Dolmella, A., Falivene, L. \& Caporaso, L. (2016). Chem. Eur. J. 22, 10211-10224.
Bruno, I. J., Cole, J. C., Edgington, P. R., Kessler, M., Macrae, C. F., McCabe, P., Pearson, J. \& Taylor, R. (2002). Acta Cryst. B58, 389397.

Büssing, R., Karge, B., Lippmann, P., Jones, P. G., Brönstrup, M. \& Ott, I. (2021). ChemMedChem. In the press (https://doi.org/10.1002/ cmdc.202100381).

Cavallo, G., Metrangolo, P., Milani, R., Pilati, T., Priimagi, A., Resnati, G. \& Terraneo, G. (2016). Chem. Rev. 116, 2478-2601.

Döring, C. \& Jones, P. G. (2016). Z. Anorg. Allg. Chem. 642, 930-936.

Ghosh, A. K. \& Catalano, V. J. (2009). Eur. J. Inorg. Chem. pp. 18321843.

Gil-Rubio, J., Cámara, V., Bautista, D. \& Vicente, J. (2013). Inorg. Chem. 52, 4071-4083.

Groom, C. R., Bruno, I. J., Lightfoot, M. P. \& Ward, S. C. (2016). Acta Cryst. B72, 171-179.

Holthoff, J. M., Engelage, E., Kowsari, A. B., Huber, S. M. \& Weiss, R. (2019). Chem. Eur. J. 25, 7480-7484.

Jothibasu, R., Huynh, H. V. \& Koh, L. L. (2008). J. Organomet. Chem. 693, 374-380.

Metrangelo, P. (2008). Angew. Chem. Int. Ed. 47, 6114-6127.

Mora, M., Gimeno, M. C. \& Visbal, R. (2019). Chem. Soc. Rev. 48 , 447-462.

Nahra, F., Tzouras, N. V., Collado, A. \& Nolan, S. P. (2021). Nat. Protoc. 16, 1476-1493.

Ott, I. (2020). Adv. Inorg. Chem. 75, 121-148.

Rigaku OD (2021). CrysAlis PRO. Rigaku Oxford Diffraction, Yarnton, England.

Schmidt, C., Karge, B., Misgeld, R., Prokop, A., Franke, R., Brönstrup, M. \& Ott, I. (2017b). Chem. Eur. J. 23, 1869-1880.

Schmidt, C., Karge, B., Misgeld, R., Prokop, A., Franke, R. \& Ott, I. (2017a). MedChemComm, 8, 1681-1689.

Sheldrick, G. M. (2008). Acta Cryst. A64, 112-122.

Sheldrick, G. M. (2015). Acta Cryst. C71, 3-8.

Siemens (1994). XP. Siemens Analytical X-Ray Instruments, Madison, Wisconsin, USA. 


\section{supporting information}

Acta Cryst. (2021). E77, 1249-1252［https://doi.org/10.1107/S2056989021011488]

Crystal structures of the gold NHC complex bis(4-bromo-1,3-diethylimidazol-2ylidene)gold(I) iodide and its 1:1 adduct with trans-bis(4-bromo-1,3-diethylimidazol-2-ylidene)diiodidogold(III) iodide

\section{Rolf Büssing, Ingo Ott and Peter G. Jones}

Computing details

For both structures, data collection: CrysAlis PRO (Rigaku OD, 2021); cell refinement: CrysAlis PRO (Rigaku OD, 2021); data reduction: CrysAlis PRO (Rigaku OD, 2021); program(s) used to solve structure: SHELXS (Sheldrick, 2008); program(s) used to refine structure: SHELXL2017 (Sheldrick, 2015); molecular graphics: Siemens XP (Siemens, 1994); software used to prepare material for publication: SHELXL2017 (Sheldrick, 2015).

Bis(4-bromo-1,3-diethylimidazol-2-ylidene)gold(I) iodide (3)

Crystal data

$\left[\mathrm{Au}\left(\mathrm{C}_{7} \mathrm{H}_{11} \mathrm{BrN}_{2}\right)_{2}\right] \mathrm{I}$

$M_{r}=730.04$

Triclinic, $P \overline{1}$

$a=8.4676(2) \AA$

$b=8.8248(3) \AA$

$c=14.0119(5) \AA$

$\alpha=76.374(3)^{\circ}$

$\beta=85.320(2)^{\circ}$

$\gamma=85.251(2)^{\circ}$

$V=1011.99(6) \AA^{3}$

\section{Data collection}

XtaLAB Synergy, HyPix diffractometer

Radiation source: micro-focus sealed X-ray tube Detector resolution: 10.0000 pixels $\mathrm{mm}^{-1}$

$\omega$ scans

Absorption correction: multi-scan

(CrysAlisPro; Rigaku OD, 2021)

$T_{\min }=0.751, T_{\max }=1.000$

Refinement

Refinement on $F^{2}$

Least-squares matrix: full

$R\left[F^{2}>2 \sigma\left(F^{2}\right)\right]=0.023$

$w R\left(F^{2}\right)=0.052$

$S=1.02$

9374 reflections
$Z=2$

$F(000)=672$

$D_{\mathrm{x}}=2.396 \mathrm{Mg} \mathrm{m}^{-3}$

Mo $K \alpha$ radiation, $\lambda=0.71073 \AA$

Cell parameters from 47541 reflections

$\theta=2.7-36.4^{\circ}$

$\mu=12.74 \mathrm{~mm}^{-1}$

$T=100 \mathrm{~K}$

Block, colourless

$0.09 \times 0.06 \times 0.05 \mathrm{~mm}$

84327 measured reflections

9374 independent reflections

8185 reflections with $I>2 \sigma(I)$

$R_{\text {int }}=0.043$

$\theta_{\text {max }}=36.7^{\circ}, \theta_{\min }=2.8^{\circ}$

$h=-14 \rightarrow 14$

$k=-14 \rightarrow 14$

$l=-22 \rightarrow 22$

203 parameters

0 restraints

Hydrogen site location: inferred from neighbouring sites

$\mathrm{H}$-atom parameters constrained 
$w=1 /\left[\sigma^{2}\left(F_{\mathrm{o}}^{2}\right)+(0.0219 P)^{2}+1.6441 P\right]$

where $P=\left(F_{\mathrm{o}}^{2}+2 F_{\mathrm{c}}^{2}\right) / 3$

$(\Delta / \sigma)_{\max }=0.002$

$$
\Delta \rho_{\max }=1.53 \mathrm{e} \AA^{-3}
$$$$
\Delta \rho_{\min }=-2.02{\mathrm{e} \AA^{-3}}^{-3}
$$

\section{Special details}

Geometry. All esds (except the esd in the dihedral angle between two 1.s. planes) are estimated using the full covariance matrix. The cell esds are taken into account individually in the estimation of esds in distances, angles and torsion angles; correlations between esds in cell parameters are only used when they are defined by crystal symmetry. An approximate (isotropic) treatment of cell esds is used for estimating esds involving l.s. planes.

Fractional atomic coordinates and isotropic or equivalent isotropic displacement parameters $\left(\AA^{2}\right)$

\begin{tabular}{|c|c|c|c|c|}
\hline & $x$ & $y$ & $z$ & $U_{\text {iso }} * / U_{\text {eq }}$ \\
\hline Aul & $0.46224(2)$ & $0.71713(2)$ & $0.23616(2)$ & $0.01505(2)$ \\
\hline I1 & $0.25568(2)$ & -0.12579 (2) & $0.72649(2)$ & $0.01947(3)$ \\
\hline $\mathrm{Br} 1$ & $0.90742(3)$ & $0.85910(3)$ & -0.11585 (2) & $0.01837(4)$ \\
\hline $\mathrm{Br} 2$ & $0.22321(3)$ & $0.27369(3)$ & $0.58678(2)$ & $0.02294(5)$ \\
\hline N1 & $0.7026(2)$ & $0.8013(2)$ & $0.06168(14)$ & $0.0144(3)$ \\
\hline N2 & $0.4687(2)$ & $0.7981(2)$ & $0.01341(14)$ & $0.0151(3)$ \\
\hline N3 & $0.3414(2)$ & $0.4840(2)$ & $0.41402(14)$ & $0.0158(3)$ \\
\hline N4 & $0.3267(2)$ & $0.7110(2)$ & $0.44721(14)$ & $0.0168(3)$ \\
\hline $\mathrm{C} 1$ & $0.5490(3)$ & $0.7807(3)$ & $0.09487(17)$ & $0.0152(4)$ \\
\hline $\mathrm{C} 2$ & $0.7156(3)$ & $0.8303(3)$ & $-0.03991(16)$ & $0.0150(4)$ \\
\hline $\mathrm{C} 3$ & $0.5681(3)$ & 0.8289 & $-0.07101(17)$ & $0.0159(4)$ \\
\hline H3 & 0.539432 & 0.845625 & -0.137005 & $0.019^{*}$ \\
\hline $\mathrm{C} 4$ & $0.8329(3)$ & $0.7876(3)$ & $0.12682(17)$ & $0.0190(4)$ \\
\hline $\mathrm{H} 4 \mathrm{~A}$ & 0.796328 & 0.835665 & 0.182530 & $0.023^{*}$ \\
\hline H4B & 0.922392 & 0.845773 & 0.090037 & $0.023^{*}$ \\
\hline $\mathrm{C} 5$ & $0.8904(3)$ & $0.6186(3)$ & $0.1667(2)$ & $0.0268(5)$ \\
\hline $\mathrm{H} 5 \mathrm{~A}$ & 0.802277 & 0.560658 & 0.203377 & $0.040^{*}$ \\
\hline $\mathrm{H} 5 \mathrm{~B}$ & 0.975985 & 0.614518 & 0.210455 & $0.040 *$ \\
\hline $\mathrm{H} 5 \mathrm{C}$ & 0.930249 & 0.571616 & 0.111832 & $0.040^{*}$ \\
\hline C6 & $0.3003(3)$ & 0.7693 & $0.01381(19)$ & $0.0190(4)$ \\
\hline H6A & 0.251033 & 0.845711 & -0.041004 & $0.023^{*}$ \\
\hline H6B & 0.244648 & 0.783981 & 0.076250 & $0.023^{*}$ \\
\hline $\mathrm{C} 7$ & $0.2815(3)$ & $0.6044(3)$ & $0.0029(2)$ & $0.0253(5)$ \\
\hline $\mathrm{H} 7 \mathrm{~A}$ & 0.335813 & 0.590123 & -0.059152 & $0.038 *$ \\
\hline H7B & 0.168425 & 0.588003 & 0.002906 & $0.038 *$ \\
\hline $\mathrm{H} 7 \mathrm{C}$ & 0.328132 & 0.528699 & 0.057982 & $0.038 *$ \\
\hline $\mathrm{C} 8$ & $0.3723(3)$ & $0.6350(3)$ & $0.37528(17)$ & $0.0164(4)$ \\
\hline C9 & 0.2739 & $0.4672(3)$ & $0.50878(17)$ & $0.0175(4)$ \\
\hline $\mathrm{C} 10$ & $0.2644(3)$ & $0.6105(3)$ & $0.53014(17)$ & $0.0190(4)$ \\
\hline H10 & 0.223233 & 0.636119 & 0.589902 & $0.023^{*}$ \\
\hline $\mathrm{C} 11$ & $0.3707(3)$ & 0.3610 & $0.35936(19)$ & $0.0208(4)$ \\
\hline H11A & 0.380137 & 0.258081 & 0.406376 & $0.025^{*}$ \\
\hline H11B & 0.472452 & 0.376079 & 0.319289 & $0.025^{*}$ \\
\hline C12 & $0.2385(4)$ & $0.3624(3)$ & $0.2928(2)$ & $0.0292(6)$ \\
\hline $\mathrm{H} 12 \mathrm{~A}$ & 0.137555 & 0.348228 & 0.332223 & $0.044 *$ \\
\hline $\mathrm{H} 12 \mathrm{~B}$ & 0.260413 & 0.277190 & 0.258600 & $0.044 *$ \\
\hline
\end{tabular}




\begin{tabular}{lllll}
$\mathrm{H} 12 \mathrm{C}$ & 0.231993 & 0.462511 & 0.244325 & $0.044^{*}$ \\
$\mathrm{C} 13$ & $0.3265(3)$ & $0.8808(3)$ & $0.43601(19)$ & $0.0204(4)$ \\
$\mathrm{H} 13 \mathrm{~A}$ & 0.407523 & 0.923764 & 0.383801 & $0.024^{*}$ \\
$\mathrm{H} 13 \mathrm{~B}$ & 0.354381 & 0.903198 & 0.498325 & $0.024^{*}$ \\
$\mathrm{C} 14$ & $0.1657(4)$ & $0.9582(3)$ & $0.4096(3)$ & $0.0367(7)$ \\
$\mathrm{H} 14 \mathrm{~A}$ & 0.140898 & 0.940989 & 0.346040 & $0.055^{*}$ \\
$\mathrm{H} 14 \mathrm{~B}$ & 0.166327 & 1.070607 & 0.405280 & $0.055^{*}$ \\
$\mathrm{H} 14 \mathrm{C}$ & 0.085156 & 0.913349 & 0.460390 & $0.055^{*}$ \\
\hline
\end{tabular}

Atomic displacement parameters $\left(\AA^{2}\right)$

\begin{tabular}{lllllll}
\hline & $U^{11}$ & $U^{22}$ & $U^{33}$ & $U^{12}$ & $U^{13}$ & $U^{23}$ \\
\hline Au1 & $0.01531(4)$ & $0.01589(4)$ & $0.01371(4)$ & $-0.00177(3)$ & $0.00196(3)$ & $-0.00363(3)$ \\
I1 & $0.02119(7)$ & $0.02104(7)$ & $0.01555(6)$ & $0.00120(5)$ & $0.00096(5)$ & $-0.00454(5)$ \\
Br1 & $0.01523(10)$ & $0.02506(11)$ & $0.01603(9)$ & $-0.00645(8)$ & $0.00254(7)$ & $-0.00658(8)$ \\
Br2 & $0.03080(13)$ & $0.02037(11)$ & $0.01701(10)$ & $-0.00987(9)$ & $-0.00213(9)$ & $-0.00004(8)$ \\
N1 & $0.0136(8)$ & $0.0164(8)$ & $0.0137(8)$ & $-0.0021(6)$ & $0.0003(6)$ & $-0.0045(6)$ \\
N2 & $0.0129(8)$ & $0.0163(8)$ & $0.0155(8)$ & $-0.0007(6)$ & $0.0000(6)$ & $-0.0031(7)$ \\
N3 & $0.0170(8)$ & $0.0159(8)$ & $0.0149(8)$ & $-0.0017(7)$ & $-0.0007(6)$ & $-0.0040(7)$ \\
N4 & $0.0204(9)$ & $0.0153(8)$ & $0.0148(8)$ & $-0.0032(7)$ & $-0.0003(7)$ & $-0.0035(7)$ \\
C1 & $0.0139(9)$ & $0.0155(9)$ & $0.0165(9)$ & $-0.0023(7)$ & $0.0017(7)$ & $-0.0046(7)$ \\
C2 & $0.0152(9)$ & $0.0172(9)$ & $0.0128(8)$ & $-0.0035(7)$ & $0.0011(7)$ & $-0.0038(7)$ \\
C3 & $0.0149(9)$ & $0.0178(10)$ & $0.0153(9)$ & $-0.0021(7)$ & $0.0001(7)$ & $-0.0045(8)$ \\
C4 & $0.0176(10)$ & $0.0256(11)$ & $0.0157(9)$ & $-0.0056(8)$ & $-0.0020(8)$ & $-0.0070(8)$ \\
C5 & $0.0241(12)$ & $0.0309(14)$ & $0.0253(12)$ & $0.0050(10)$ & $-0.0074(10)$ & $-0.0065(10)$ \\
C6 & $0.0119(9)$ & $0.0231(11)$ & $0.0217(10)$ & $-0.0001(8)$ & $-0.0017(8)$ & $-0.0049(9)$ \\
C7 & $0.0201(11)$ & $0.0243(12)$ & $0.0330(13)$ & $-0.0066(9)$ & $-0.0003(10)$ & $-0.0081(10)$ \\
C8 & $0.0153(9)$ & $0.0167(9)$ & $0.0169(9)$ & $-0.0016(7)$ & $-0.0004(7)$ & $-0.0036(8)$ \\
C9 & $0.0211(10)$ & $0.0171(10)$ & $0.0136(9)$ & $-0.0045(8)$ & $-0.0012(8)$ & $-0.0011(8)$ \\
C10 & $0.0218(11)$ & $0.0199(10)$ & $0.0151(9)$ & $-0.0047(8)$ & $0.0014(8)$ & $-0.0037(8)$ \\
C11 & $0.0251(12)$ & $0.0174(10)$ & $0.0212(11)$ & $-0.0008(9)$ & $-0.0005(9)$ & $-0.0075(9)$ \\
C12 & $0.0391(16)$ & $0.0252(13)$ & $0.0264(13)$ & $-0.0052(11)$ & $-0.0088(11)$ & $-0.0085(10)$ \\
C13 & $0.0271(12)$ & $0.0143(10)$ & $0.0200(10)$ & $-0.0054(8)$ & $0.0024(9)$ & $-0.0042(8)$ \\
C14 & $0.0328(16)$ & $0.0179(12)$ & $0.056(2)$ & $0.0019(11)$ & $-0.0004(14)$ & $-0.0034(13)$ \\
& & & & & & \\
\hline & & & & & & \\
\hline
\end{tabular}

Geometric parameters $\left(\AA,{ }^{\circ}\right)$

\begin{tabular}{llll}
\hline $\mathrm{Au} 1-\mathrm{C} 1$ & $2.020(2)$ & $\mathrm{C} 13-\mathrm{C} 14$ & $1.506(4)$ \\
$\mathrm{Au} 1-\mathrm{C} 8$ & $2.022(2)$ & $\mathrm{C} 3-\mathrm{H} 3$ & 0.9500 \\
$\mathrm{I} 1-\mathrm{Br} 1^{\mathrm{i}}$ & $3.5294(3)$ & $\mathrm{C} 4-\mathrm{H} 4 \mathrm{~A}$ & 0.9900 \\
$\mathrm{I} 1-\mathrm{Br} 2$ & $3.6072(3)$ & $\mathrm{C} 4-\mathrm{H} 4 \mathrm{~B}$ & 0.9900 \\
$\mathrm{Au} 1-\mathrm{Br} 2^{\mathrm{ii}}$ & $3.8033(3)$ & $\mathrm{C} 5-\mathrm{H} 5 \mathrm{~A}$ & 0.9800 \\
$\mathrm{Br} 1-\mathrm{C} 2$ & $1.869(2)$ & $\mathrm{C} 5-\mathrm{H} 5 \mathrm{~B}$ & 0.9800 \\
$\mathrm{Br} 2-\mathrm{C} 9$ & $1.861(2)$ & $\mathrm{C} 5-\mathrm{H} 5 \mathrm{C}$ & 0.9800 \\
$\mathrm{~N} 1-\mathrm{C} 1$ & $1.357(3)$ & $\mathrm{C} 6-\mathrm{H} 6 \mathrm{~A}$ & 0.9900 \\
$\mathrm{~N} 1-\mathrm{C} 2$ & $1.382(3)$ & $\mathrm{C} 6-\mathrm{H} 6 \mathrm{~B}$ & 0.9900 \\
$\mathrm{~N} 1-\mathrm{C} 4$ & $1.468(3)$ & $\mathrm{C} 7-\mathrm{H} 7 \mathrm{~A}$ & 0.9800 \\
$\mathrm{~N} 2-\mathrm{C} 1$ & $1.348(3)$ & $\mathrm{C} 7-\mathrm{H} 7 \mathrm{~B}$ & 0.9800
\end{tabular}




\begin{tabular}{|c|c|c|c|}
\hline $\mathrm{N} 2-\mathrm{C} 3$ & $1.381(3)$ & $\mathrm{C} 7-\mathrm{H} 7 \mathrm{C}$ & 0.9800 \\
\hline $\mathrm{N} 2-\mathrm{C} 6$ & $1.468(3)$ & $\mathrm{C} 10-\mathrm{H} 10$ & 0.9500 \\
\hline N3- C8 & $1.354(3)$ & $\mathrm{C} 11-\mathrm{H} 11 \mathrm{~A}$ & 0.9900 \\
\hline N3-C9 & $1.382(3)$ & C11-H11B & 0.9900 \\
\hline N3-C11 & $1.464(3)$ & $\mathrm{C} 12-\mathrm{H} 12 \mathrm{~A}$ & 0.9800 \\
\hline $\mathrm{N} 4-\mathrm{C} 8$ & $1.351(3)$ & $\mathrm{C} 12-\mathrm{H} 12 \mathrm{~B}$ & 0.9800 \\
\hline $\mathrm{N} 4-\mathrm{C} 10$ & $1.381(3)$ & $\mathrm{C} 12-\mathrm{H} 12 \mathrm{C}$ & 0.9800 \\
\hline $\mathrm{N} 4-\mathrm{C} 13$ & $1.469(3)$ & $\mathrm{C} 13-\mathrm{H} 13 \mathrm{~A}$ & 0.9900 \\
\hline $\mathrm{C} 2-\mathrm{C} 3$ & $1.357(3)$ & $\mathrm{C} 13-\mathrm{H} 13 \mathrm{~B}$ & 0.9900 \\
\hline $\mathrm{C} 4-\mathrm{C} 5$ & $1.518(4)$ & $\mathrm{C} 14-\mathrm{H} 14 \mathrm{~A}$ & 0.9800 \\
\hline $\mathrm{C} 6-\mathrm{C} 7$ & $1.521(4)$ & C14-H14B & 0.9800 \\
\hline $\mathrm{C} 9-\mathrm{C} 10$ & $1.361(3)$ & $\mathrm{C} 14-\mathrm{H} 14 \mathrm{C}$ & 0.9800 \\
\hline $\mathrm{C} 11-\mathrm{C} 12$ & $1.512(4)$ & & \\
\hline $\mathrm{C} 1-\mathrm{Au} 1-\mathrm{C} 8$ & $174.97(9)$ & $\mathrm{C} 4-\mathrm{C} 5-\mathrm{H} 5 \mathrm{~A}$ & 109.5 \\
\hline $\mathrm{Br} 1-\mathrm{I} 1-\mathrm{Br} 2$ & $101.436(8)$ & $\mathrm{C} 4-\mathrm{C} 5-\mathrm{H} 5 \mathrm{~B}$ & 109.5 \\
\hline $\mathrm{C} 2-\mathrm{Br} 1-\mathrm{I} 1^{\mathrm{iii}}$ & $172.43(7)$ & $\mathrm{H} 5 \mathrm{~A}-\mathrm{C} 5-\mathrm{H} 5 \mathrm{~B}$ & 109.5 \\
\hline $\mathrm{C} 9-\mathrm{Br} 2-\mathrm{I} 1$ & $162.21(8)$ & $\mathrm{C} 4-\mathrm{C} 5-\mathrm{H} 5 \mathrm{C}$ & 109.5 \\
\hline $\mathrm{C} 1-\mathrm{N} 1-\mathrm{C} 2$ & $109.82(19)$ & $\mathrm{H} 5 \mathrm{~A}-\mathrm{C} 5-\mathrm{H} 5 \mathrm{C}$ & 109.5 \\
\hline $\mathrm{C} 1-\mathrm{N} 1-\mathrm{C} 4$ & $123.48(19)$ & $\mathrm{H} 5 \mathrm{~B}-\mathrm{C} 5-\mathrm{H} 5 \mathrm{C}$ & 109.5 \\
\hline $\mathrm{C} 2-\mathrm{N} 1-\mathrm{C} 4$ & $126.65(19)$ & $\mathrm{N} 2-\mathrm{C} 6-\mathrm{H} 6 \mathrm{~A}$ & 109.5 \\
\hline $\mathrm{C} 1-\mathrm{N} 2-\mathrm{C} 3$ & $111.61(19)$ & $\mathrm{C} 7-\mathrm{C} 6-\mathrm{H} 6 \mathrm{~A}$ & 109.5 \\
\hline $\mathrm{C} 1-\mathrm{N} 2-\mathrm{C} 6$ & $124.54(19)$ & $\mathrm{N} 2-\mathrm{C} 6-\mathrm{H} 6 \mathrm{~B}$ & 109.5 \\
\hline $\mathrm{C} 3-\mathrm{N} 2-\mathrm{C} 6$ & $123.46(19)$ & $\mathrm{C} 7-\mathrm{C} 6-\mathrm{H} 6 \mathrm{~B}$ & 109.5 \\
\hline $\mathrm{C} 8-\mathrm{N} 3-\mathrm{C} 9$ & $110.15(19)$ & $\mathrm{H} 6 \mathrm{~A}-\mathrm{C} 6-\mathrm{H} 6 \mathrm{~B}$ & 108.1 \\
\hline $\mathrm{C} 8-\mathrm{N} 3-\mathrm{C} 11$ & $123.4(2)$ & $\mathrm{C} 6-\mathrm{C} 7-\mathrm{H} 7 \mathrm{~A}$ & 109.5 \\
\hline $\mathrm{C} 9-\mathrm{N} 3-\mathrm{C} 11$ & $126.4(2)$ & $\mathrm{C} 6-\mathrm{C} 7-\mathrm{H} 7 \mathrm{~B}$ & 109.5 \\
\hline $\mathrm{C} 8-\mathrm{N} 4-\mathrm{C} 10$ & $111.1(2)$ & $\mathrm{H} 7 \mathrm{~A}-\mathrm{C} 7-\mathrm{H} 7 \mathrm{~B}$ & 109.5 \\
\hline $\mathrm{C} 8-\mathrm{N} 4-\mathrm{C} 13$ & $124.9(2)$ & $\mathrm{C} 6-\mathrm{C} 7-\mathrm{H} 7 \mathrm{C}$ & 109.5 \\
\hline $\mathrm{C} 10-\mathrm{N} 4-\mathrm{C} 13$ & $123.7(2)$ & $\mathrm{H} 7 \mathrm{~A}-\mathrm{C} 7-\mathrm{H} 7 \mathrm{C}$ & 109.5 \\
\hline $\mathrm{N} 2-\mathrm{C} 1-\mathrm{N} 1$ & $105.25(19)$ & $\mathrm{H} 7 \mathrm{~B}-\mathrm{C} 7-\mathrm{H} 7 \mathrm{C}$ & 109.5 \\
\hline $\mathrm{N} 2-\mathrm{C} 1-\mathrm{Au} 1$ & $127.11(16)$ & $\mathrm{C} 9-\mathrm{C} 10-\mathrm{H} 10$ & 127.0 \\
\hline $\mathrm{N} 1-\mathrm{C} 1-\mathrm{Au} 1$ & $127.41(17)$ & $\mathrm{N} 4-\mathrm{C} 10-\mathrm{H} 10$ & 127.0 \\
\hline $\mathrm{C} 3-\mathrm{C} 2-\mathrm{N} 1$ & $107.77(19)$ & $\mathrm{N} 3-\mathrm{C} 11-\mathrm{H} 11 \mathrm{~A}$ & 109.3 \\
\hline $\mathrm{C} 3-\mathrm{C} 2-\mathrm{Br} 1$ & $128.16(17)$ & $\mathrm{C} 12-\mathrm{C} 11-\mathrm{H} 11 \mathrm{~A}$ & 109.3 \\
\hline $\mathrm{N} 1-\mathrm{C} 2-\mathrm{Br} 1$ & $124.05(17)$ & N3-C11-H11B & 109.3 \\
\hline $\mathrm{C} 2-\mathrm{C} 3-\mathrm{N} 2$ & $105.6(2)$ & $\mathrm{C} 12-\mathrm{C} 11-\mathrm{H} 11 \mathrm{~B}$ & 109.3 \\
\hline $\mathrm{N} 1-\mathrm{C} 4-\mathrm{C} 5$ & $112.1(2)$ & $\mathrm{H} 11 \mathrm{~A}-\mathrm{C} 11-\mathrm{H} 11 \mathrm{~B}$ & 108.0 \\
\hline $\mathrm{N} 2-\mathrm{C} 6-\mathrm{C} 7$ & $110.9(2)$ & $\mathrm{C} 11-\mathrm{C} 12-\mathrm{H} 12 \mathrm{~A}$ & 109.5 \\
\hline $\mathrm{N} 4-\mathrm{C} 8-\mathrm{N} 3$ & $105.41(19)$ & $\mathrm{C} 11-\mathrm{C} 12-\mathrm{H} 12 \mathrm{~B}$ & 109.5 \\
\hline $\mathrm{N} 4-\mathrm{C} 8-\mathrm{Au} 1$ & $130.27(17)$ & $\mathrm{H} 12 \mathrm{~A}-\mathrm{C} 12-\mathrm{H} 12 \mathrm{~B}$ & 109.5 \\
\hline $\mathrm{N} 3-\mathrm{C} 8-\mathrm{Au} 1$ & $124.30(17)$ & $\mathrm{C} 11-\mathrm{C} 12-\mathrm{H} 12 \mathrm{C}$ & 109.5 \\
\hline $\mathrm{C} 10-\mathrm{C} 9-\mathrm{N} 3$ & $107.3(2)$ & $\mathrm{H} 12 \mathrm{~A}-\mathrm{C} 12-\mathrm{H} 12 \mathrm{C}$ & 109.5 \\
\hline $\mathrm{C} 10-\mathrm{C} 9-\mathrm{Br} 2$ & $130.48(18)$ & $\mathrm{H} 12 \mathrm{~B}-\mathrm{C} 12-\mathrm{H} 12 \mathrm{C}$ & 109.5 \\
\hline $\mathrm{N} 3-\mathrm{C} 9-\mathrm{Br} 2$ & $122.09(17)$ & $\mathrm{N} 4-\mathrm{C} 13-\mathrm{H} 13 \mathrm{~A}$ & 109.5 \\
\hline $\mathrm{C} 9-\mathrm{C} 10-\mathrm{N} 4$ & $106.0(2)$ & $\mathrm{C} 14-\mathrm{C} 13-\mathrm{H} 13 \mathrm{~A}$ & 109.5 \\
\hline $\mathrm{N} 3-\mathrm{C} 11-\mathrm{C} 12$ & $111.5(2)$ & N4-C13-H13B & 109.5 \\
\hline $\mathrm{N} 4-\mathrm{C} 13-\mathrm{C} 14$ & $110.6(2)$ & $\mathrm{C} 14-\mathrm{C} 13-\mathrm{H} 13 \mathrm{~B}$ & 109.5 \\
\hline
\end{tabular}




\begin{tabular}{|c|c|c|c|}
\hline $\mathrm{C} 2-\mathrm{C} 3-\mathrm{H} 3$ & 127.2 & $\mathrm{H} 13 \mathrm{~A}-\mathrm{C} 13-\mathrm{H} 13 \mathrm{~B}$ & 108.1 \\
\hline $\mathrm{N} 2-\mathrm{C} 3-\mathrm{H} 3$ & 127.2 & $\mathrm{C} 13-\mathrm{C} 14-\mathrm{H} 14 \mathrm{~A}$ & 109.5 \\
\hline $\mathrm{N} 1-\mathrm{C} 4-\mathrm{H} 4 \mathrm{~A}$ & 109.2 & $\mathrm{C} 13-\mathrm{C} 14-\mathrm{H} 14 \mathrm{~B}$ & 109.5 \\
\hline $\mathrm{C} 5-\mathrm{C} 4-\mathrm{H} 4 \mathrm{~A}$ & 109.2 & $\mathrm{H} 14 \mathrm{~A}-\mathrm{C} 14-\mathrm{H} 14 \mathrm{~B}$ & 109.5 \\
\hline $\mathrm{N} 1-\mathrm{C} 4-\mathrm{H} 4 \mathrm{~B}$ & 109.2 & $\mathrm{C} 13-\mathrm{C} 14-\mathrm{H} 14 \mathrm{C}$ & 109.5 \\
\hline $\mathrm{C} 5-\mathrm{C} 4-\mathrm{H} 4 \mathrm{~B}$ & 109.2 & $\mathrm{H} 14 \mathrm{~A}-\mathrm{C} 14-\mathrm{H} 14 \mathrm{C}$ & 109.5 \\
\hline $\mathrm{H} 4 \mathrm{~A}-\mathrm{C} 4-\mathrm{H} 4 \mathrm{~B}$ & 107.9 & $\mathrm{H} 14 \mathrm{~B}-\mathrm{C} 14-\mathrm{H} 14 \mathrm{C}$ & 109.5 \\
\hline $\mathrm{C} 3-\mathrm{N} 2-\mathrm{C} 1-\mathrm{N} 1$ & $0.2(3)$ & $\mathrm{C} 13-\mathrm{N} 4-\mathrm{C} 8-\mathrm{N} 3$ & $-175.0(2)$ \\
\hline $\mathrm{C} 6-\mathrm{N} 2-\mathrm{C} 1-\mathrm{N} 1$ & $173.2(2)$ & $\mathrm{C} 10-\mathrm{N} 4-\mathrm{C} 8-\mathrm{Au} 1$ & $177.04(18)$ \\
\hline $\mathrm{C} 3-\mathrm{N} 2-\mathrm{C} 1-\mathrm{Au} 1$ & $-174.55(17)$ & $\mathrm{C} 13-\mathrm{N} 4-\mathrm{C} 8-\mathrm{Au} 1$ & $3.3(4)$ \\
\hline $\mathrm{C} 6-\mathrm{N} 2-\mathrm{C} 1-\mathrm{Au} 1$ & $-1.5(3)$ & $\mathrm{C} 9-\mathrm{N} 3-\mathrm{C} 8-\mathrm{N} 4$ & $1.3(3)$ \\
\hline $\mathrm{C} 2-\mathrm{N} 1-\mathrm{C} 1-\mathrm{N} 2$ & $-0.3(2)$ & $\mathrm{C} 11-\mathrm{N} 3-\mathrm{C} 8-\mathrm{N} 4$ & $178.8(2)$ \\
\hline $\mathrm{C} 4-\mathrm{N} 1-\mathrm{C} 1-\mathrm{N} 2$ & $-178.1(2)$ & $\mathrm{C} 9-\mathrm{N} 3-\mathrm{C} 8-\mathrm{Au} 1$ & $-177.17(17)$ \\
\hline $\mathrm{C} 2-\mathrm{N} 1-\mathrm{C} 1-\mathrm{Au} 1$ & $174.36(16)$ & $\mathrm{C} 11-\mathrm{N} 3-\mathrm{C} 8-\mathrm{Au} 1$ & $0.4(3)$ \\
\hline $\mathrm{C} 4-\mathrm{N} 1-\mathrm{C} 1-\mathrm{Au} 1$ & $-3.4(3)$ & $\mathrm{C} 8-\mathrm{N} 3-\mathrm{C} 9-\mathrm{C} 10$ & $-0.8(3)$ \\
\hline $\mathrm{C} 1-\mathrm{N} 1-\mathrm{C} 2-\mathrm{C} 3$ & $0.4(3)$ & $\mathrm{C} 11-\mathrm{N} 3-\mathrm{C} 9-\mathrm{C} 10$ & $-178.3(2)$ \\
\hline $\mathrm{C} 4-\mathrm{N} 1-\mathrm{C} 2-\mathrm{C} 3$ & $178.1(2)$ & $\mathrm{C} 8-\mathrm{N} 3-\mathrm{C} 9-\mathrm{Br} 2$ & $-177.42(17)$ \\
\hline $\mathrm{C} 1-\mathrm{N} 1-\mathrm{C} 2-\mathrm{Br} 1$ & $-177.92(17)$ & $\mathrm{C} 11-\mathrm{N} 3-\mathrm{C} 9-\mathrm{Br} 2$ & $5.1(3)$ \\
\hline $\mathrm{C} 4-\mathrm{N} 1-\mathrm{C} 2-\mathrm{Br} 1$ & $-0.2(3)$ & $\mathrm{I} 1-\mathrm{Br} 2-\mathrm{C} 9-\mathrm{C} 10$ & $-114.8(3)$ \\
\hline $\mathrm{N} 1-\mathrm{C} 2-\mathrm{C} 3-\mathrm{N} 2$ & $-0.3(3)$ & $\mathrm{I} 1-\mathrm{Br} 2-\mathrm{C} 9-\mathrm{N} 3$ & $61.0(4)$ \\
\hline $\mathrm{Br} 1-\mathrm{C} 2-\mathrm{C} 3-\mathrm{N} 2$ & $177.95(17)$ & $\mathrm{N} 3-\mathrm{C} 9-\mathrm{C} 10-\mathrm{N} 4$ & $0.0(3)$ \\
\hline $\mathrm{C} 1-\mathrm{N} 2-\mathrm{C} 3-\mathrm{C} 2$ & $0.1(3)$ & $\mathrm{Br} 2-\mathrm{C} 9-\mathrm{C} 10-\mathrm{N} 4$ & $176.24(19)$ \\
\hline $\mathrm{C} 6-\mathrm{N} 2-\mathrm{C} 3-\mathrm{C} 2$ & $-173.1(2)$ & $\mathrm{C} 8-\mathrm{N} 4-\mathrm{C} 10-\mathrm{C} 9$ & $0.8(3)$ \\
\hline $\mathrm{C} 1-\mathrm{N} 1-\mathrm{C} 4-\mathrm{C} 5$ & $81.0(3)$ & $\mathrm{C} 13-\mathrm{N} 4-\mathrm{C} 10-\mathrm{C} 9$ & $174.6(2)$ \\
\hline $\mathrm{C} 2-\mathrm{N} 1-\mathrm{C} 4-\mathrm{C} 5$ & $-96.4(3)$ & $\mathrm{C} 8-\mathrm{N} 3-\mathrm{C} 11-\mathrm{C} 12$ & $-80.8(3)$ \\
\hline $\mathrm{C} 1-\mathrm{N} 2-\mathrm{C} 6-\mathrm{C} 7$ & $-94.9(3)$ & $\mathrm{C} 9-\mathrm{N} 3-\mathrm{C} 11-\mathrm{C} 12$ & $96.4(3)$ \\
\hline $\mathrm{C} 3-\mathrm{N} 2-\mathrm{C} 6-\mathrm{C} 7$ & $77.4(3)$ & $\mathrm{C} 8-\mathrm{N} 4-\mathrm{C} 13-\mathrm{C} 14$ & $94.0(3)$ \\
\hline $\mathrm{C} 10-\mathrm{N} 4-\mathrm{C} 8-\mathrm{N} 3$ & $-1.3(3)$ & $\mathrm{C} 10-\mathrm{N} 4-\mathrm{C} 13-\mathrm{C} 14$ & $-79.0(3)$ \\
\hline
\end{tabular}

Symmetry codes: (i) $x-1, y-1, z+1$; (ii) $-x+1,-y+1,-z+1$; (iii) $x+1, y+1, z-1$.

Hydrogen-bond geometry $\left(A,{ }^{\circ}\right)$

\begin{tabular}{lllll}
\hline$D-\mathrm{H} \cdots A$ & $D-\mathrm{H}$ & $\mathrm{H} \cdots A$ & $D \cdots A$ & $D-\mathrm{H} \cdots A$ \\
\hline $\mathrm{C} 3-\mathrm{H} 3 \cdots \mathrm{I} 1^{\mathrm{iv}}$ & 0.95 & 3.15 & $3.966(2)$ & 145 \\
$\mathrm{C} 4-\mathrm{H} 4 A \cdots \mathrm{I} 1^{\mathrm{ii}}$ & 0.99 & 3.10 & $3.995(3)$ & 151 \\
$\mathrm{C} 6-\mathrm{H} 6 A \cdots \mathrm{I} 1^{\text {iv }}$ & 0.99 & 3.21 & $3.955(3)$ & 134 \\
$\mathrm{C} 10-\mathrm{H} 10 \cdots \mathrm{I} 1^{\mathrm{v}}$ & 0.95 & 3.20 & $3.993(2)$ & 142 \\
$\mathrm{C} 11-\mathrm{H} 11 A \cdots \mathrm{Br} 2$ & 0.99 & 2.79 & $3.265(3)$ & 110 \\
$\mathrm{C} 11-\mathrm{H} 11 B \cdots \mathrm{I} 1^{\mathrm{vi}}$ & 0.99 & 3.19 & $3.903(3)$ & 130 \\
$\mathrm{C} 12-\mathrm{H} 12 B \cdots \mathrm{Br} 1^{\mathrm{vii}}$ & 0.98 & 3.06 & $3.831(3)$ & 137 \\
$\mathrm{C} 13-\mathrm{H} 13 B \cdots \mathrm{I} 1^{\mathrm{v}}$ & 0.99 & 3.19 & $4.053(3)$ & 146 \\
\hline
\end{tabular}

Symmetry codes: (ii) $-x+1,-y+1,-z+1$; (iv) $x, y+1, z-1$; (v) $x, y+1, z$; (vi) $-x+1,-y,-z+1$; (vii) $-x+1,-y+1,-z$. 
Bis(4-bromo-1,3-diethylimidazol-2-ylidene)gold(I) trans-bis(4-bromo-1,3-diethyl-imidazol-2-

ylidene)diiodidogold(III) diiodide (4)

\section{Crystal data}

$\left[\mathrm{Au}\left(\mathrm{C}_{7} \mathrm{H}_{11} \mathrm{BrN}_{2}\right)_{2}\right]\left[\mathrm{AuI}_{2}\left(\mathrm{C}_{7} \mathrm{H}_{11} \mathrm{BrN}_{2}\right)_{2}\right] \mathrm{I}_{2}$

$M_{r}=1713.88$

Triclinic, $P \overline{1}$

$a=8.0245$ (4) $\AA$

$b=8.5782(3) \AA$

$c=15.9814(6) \AA$

$\alpha=91.228(3)^{\circ}$

$\beta=96.517(4)^{\circ}$

$\gamma=92.255(4)^{\circ}$

$V=1091.77(8) \AA^{3}$

\section{Data collection}

XtaLAB Synergy, HyPix diffractometer

Radiation source: micro-focus sealed X-ray tube Detector resolution: 10.0000 pixels $\mathrm{mm}^{-1}$ $\omega$ scans

Absorption correction: multi-scan

(CrysAlisPro; Rigaku OD, 2021)

$T_{\min }=0.703, T_{\max }=1.000$

\section{Refinement}

Refinement on $F^{2}$

Least-squares matrix: full

$R\left[F^{2}>2 \sigma\left(F^{2}\right)\right]=0.039$

$w R\left(F^{2}\right)=0.105$

$S=1.06$

6378 reflections

215 parameters

0 restraints

$$
\begin{aligned}
& Z=1 \\
& F(000)=778 \\
& D_{\mathrm{x}}=2.607 \mathrm{Mg} \mathrm{m}^{-3}
\end{aligned}
$$

Mo $K \alpha$ radiation, $\lambda=0.71073 \AA$

Cell parameters from 24546 reflections

$\theta=2.6-34.1^{\circ}$

$\mu=13.23 \mathrm{~mm}^{-1}$

$T=100 \mathrm{~K}$

Plate, brown

$0.08 \times 0.03 \times 0.01 \mathrm{~mm}$

Special details

61886 measured reflections 6378 independent reflections 5409 reflections with $I>2 \sigma(I)$

$R_{\text {int }}=0.048$

$\theta_{\max }=30.0^{\circ}, \theta_{\min }=2.7^{\circ}$

$h=-11 \rightarrow 11$

$k=-12 \rightarrow 12$

$l=-22 \rightarrow 22$

Hydrogen site location: inferred from neighbouring sites

$\mathrm{H}$-atom parameters constrained

$w=1 /\left[\sigma^{2}\left(F_{\mathrm{o}}^{2}\right)+(0.0494 P)^{2}+12.4607 P\right]$

where $P=\left(F_{\mathrm{o}}^{2}+2 F_{\mathrm{c}}^{2}\right) / 3$

$(\Delta / \sigma)_{\max }<0.001$

$\Delta \rho_{\max }=3.97 \mathrm{e} \AA^{-3}$

$\Delta \rho_{\min }=-2.66$ e $\AA^{-3}$

Geometry. All esds (except the esd in the dihedral angle between two 1.s. planes) are estimated using the full covariance matrix. The cell esds are taken into account individually in the estimation of esds in distances, angles and torsion angles; correlations between esds in cell parameters are only used when they are defined by crystal symmetry. An approximate (isotropic) treatment of cell esds is used for estimating esds involving 1.s. planes.

Fractional atomic coordinates and isotropic or equivalent isotropic displacement parameters $\left(\AA^{2}\right)$

\begin{tabular}{lllll}
\hline & $x$ & $y$ & $z$ & $U_{\text {iso }} * / U_{\text {eq }}$ \\
\hline Au1 & -0.500000 & -0.500000 & 0.000000 & $0.01890(8)$ \\
I1 & $-0.36292(6)$ & $-0.37952(6)$ & $-0.12916(3)$ & $0.02666(11)$ \\
Br1 & $-0.00401(10)$ & $-0.09434(9)$ & $0.21316(5)$ & $0.02965(16)$ \\
N1 & $-0.2548(7)$ & $-0.2807(7)$ & $0.1082(4)$ & $0.0227(11)$ \\
N2 & $-0.1732(7)$ & $-0.5172(7)$ & $0.1164(4)$ & $0.0218(11)$ \\
C1 & $-0.2957(9)$ & $-0.4283(8)$ & $0.0806(4)$ & $0.0199(12)$ \\
C2 & $-0.1053(9)$ & $-0.2781(8)$ & $0.1624(4)$ & $0.0236(13)$ \\
C3 & $-0.0556(9)$ & $-0.4261(8)$ & $0.1680(4)$ & $0.0242(13)$
\end{tabular}




\begin{tabular}{|c|c|c|c|c|}
\hline $\mathrm{H} 3$ & 0.041194 & -0.461233 & 0.200890 & $0.029^{*}$ \\
\hline $\mathrm{C} 4$ & $-0.3602(10)$ & $-0.1472(8)$ & $0.0908(5)$ & $0.0282(15)$ \\
\hline $\mathrm{H} 4 \mathrm{~A}$ & -0.426098 & -0.163377 & 0.034872 & $0.034^{*}$ \\
\hline H4B & -0.287389 & -0.051785 & 0.088776 & $0.034 *$ \\
\hline $\mathrm{C} 5$ & $-0.4806(13)$ & $-0.1229(11)$ & $0.1572(6)$ & $0.042(2)$ \\
\hline H5A & -0.548069 & -0.219305 & 0.162128 & $0.064^{*}$ \\
\hline H5B & -0.554816 & -0.038287 & 0.140329 & $0.064 *$ \\
\hline $\mathrm{H} 5 \mathrm{C}$ & -0.415991 & -0.095597 & 0.211660 & $0.064 *$ \\
\hline C6 & $-0.1643(9)$ & $-0.6878(8)$ & 0.1038 (4) & $0.0243(13)$ \\
\hline H6A & -0.276204 & -0.738050 & 0.107783 & $0.029 *$ \\
\hline H6B & -0.084560 & -0.728786 & 0.149017 & $0.029^{*}$ \\
\hline C7 & $-0.1087(11)$ & $-0.7291(10)$ & $0.0196(5)$ & $0.0343(17)$ \\
\hline $\mathrm{H} 7 \mathrm{~A}$ & -0.194248 & -0.699822 & -0.025403 & $0.051^{*}$ \\
\hline H7B & -0.093445 & -0.841752 & 0.015793 & $0.051^{*}$ \\
\hline $\mathrm{H} 7 \mathrm{C}$ & -0.002321 & -0.672810 & 0.013535 & $0.051^{*}$ \\
\hline $\mathrm{Au} 2$ & 1.000000 & 1.000000 & 0.500000 & $0.02101(9)$ \\
\hline $\mathrm{I} 2$ & $0.18002(6)$ & $0.24488(5)$ & $0.30519(3)$ & $0.02377(10)$ \\
\hline $\mathrm{Br} 2$ & $0.40896(9)$ & $0.59948(8)$ & $0.36840(4)$ & 0.02459 (14) \\
\hline $\mathrm{N} 1^{\prime}$ & $0.7026(7)$ & $0.7839(6)$ & $0.4369(3)$ & $0.0198(11)$ \\
\hline $\mathrm{N} 2^{\prime}$ & $0.6387(8)$ & $1.0226(6)$ & $0.4186(4)$ & $0.0219(11)$ \\
\hline $\mathrm{C} 1^{\prime}$ & $0.7658(9)$ & $0.9331(7)$ & $0.4491(4)$ & $0.0203(12)$ \\
\hline $\mathrm{C} 2^{\prime}$ & $0.5388(9)$ & $0.7821(8)$ & 0.3989 (4) & $0.0216(12)$ \\
\hline $\mathrm{C} 3^{\prime}$ & $0.4977(9)$ & $0.9322(8)$ & $0.3871(4)$ & $0.0215(12)$ \\
\hline H3' & 0.393497 & 0.968182 & 0.362203 & $0.026^{*}$ \\
\hline $\mathrm{C} 4^{\prime}$ & $0.8021(9)$ & $0.6466(8)$ & 0.4567 (4) & $0.0224(13)$ \\
\hline $\mathrm{H} 4^{\prime} 1$ & 0.725634 & 0.555349 & 0.463211 & $0.027^{*}$ \\
\hline $\mathrm{H} 4^{\prime} 2$ & 0.873672 & 0.665833 & 0.510905 & $0.027^{*}$ \\
\hline $\mathrm{C} 5^{\prime}$ & $0.9122(10)$ & $0.6105(8)$ & $0.3881(5)$ & $0.0290(15)$ \\
\hline $\mathrm{H} 5^{\prime} 1$ & 0.841801 & 0.593654 & 0.334152 & $0.044^{*}$ \\
\hline $\mathrm{H} 5^{\prime} 2$ & 0.973515 & 0.516180 & 0.402016 & $0.044^{*}$ \\
\hline $\mathrm{H} 5{ }^{\prime} 3$ & 0.992338 & 0.698354 & 0.383758 & $0.044 *$ \\
\hline $\mathrm{C} 6^{\prime}$ & $0.6523(10)$ & $1.1916(8)$ & $0.4105(5)$ & $0.0259(14)$ \\
\hline $\mathrm{H} 6^{\prime} 1$ & 0.746773 & 1.235149 & 0.450502 & $0.031^{*}$ \\
\hline $\mathrm{H} 6^{\prime} 2$ & 0.547969 & 1.238037 & 0.425063 & $0.031 *$ \\
\hline C7' & $0.6810(9)$ & $1.2344(8)$ & $0.3214(5)$ & $0.0281(15)$ \\
\hline $\mathrm{H}^{\prime}{ }^{\prime} 1$ & 0.785274 & 1.189945 & 0.307254 & $0.042 *$ \\
\hline $\mathrm{H} 7^{\prime} 2$ & 0.689663 & 1.348279 & 0.317476 & $0.042 *$ \\
\hline $\mathrm{H} 7^{\prime} 3$ & 0.586704 & 1.192742 & 0.281833 & $0.042 *$ \\
\hline
\end{tabular}

Atomic displacement parameters $\left(\AA^{2}\right)$

\begin{tabular}{lllllll}
\hline & $U^{11}$ & $U^{22}$ & $U^{33}$ & $U^{12}$ & $U^{13}$ & $U^{23}$ \\
\hline Au1 & $0.02247(17)$ & $0.01893(16)$ & $0.01489(15)$ & $-0.00122(12)$ & $0.00110(12)$ & $0.00024(12)$ \\
I1 & $0.0288(2)$ & $0.0301(2)$ & $0.0214(2)$ & $-0.00043(18)$ & $0.00418(16)$ & $0.00327(17)$ \\
Br1 & $0.0348(4)$ & $0.0248(3)$ & $0.0272(3)$ & $-0.0053(3)$ & $-0.0026(3)$ & $-0.0047(3)$ \\
N1 & $0.025(3)$ & $0.023(3)$ & $0.020(3)$ & $0.000(2)$ & $0.004(2)$ & $-0.002(2)$ \\
N2 & $0.022(3)$ & $0.021(3)$ & $0.021(3)$ & $-0.002(2)$ & $-0.001(2)$ & $-0.003(2)$ \\
C1 & $0.028(3)$ & $0.018(3)$ & $0.013(3)$ & $-0.002(2)$ & $0.002(2)$ & $-0.001(2)$
\end{tabular}




$\begin{array}{lllllll}\mathrm{C} 2 & 0.028(3) & 0.024(3) & 0.018(3) & -0.004(3) & 0.001(2) & -0.003(2) \\ \mathrm{C} 3 & 0.028(3) & 0.027(3) & 0.017(3) & 0.000(3) & 0.000(2) & -0.004(2) \\ \mathrm{C} 4 & 0.039(4) & 0.019(3) & 0.025(3) & -0.001(3) & 0.000(3) & -0.002(3) \\ \mathrm{C} 5 & 0.051(5) & 0.035(4) & 0.042(5) & 0.004(4) & 0.014(4) & -0.008(4) \\ \mathrm{C} 6 & 0.028(3) & 0.021(3) & 0.024(3) & 0.001(3) & 0.002(3) & 0.000(3) \\ \mathrm{C} 7 & 0.042(5) & 0.031(4) & 0.031(4) & 0.005(3) & 0.005(3) & -0.007(3) \\ \mathrm{Au} 2 & 0.02690(18) & 0.01581(16) & 0.01892(16) & 0.00103(12) & -0.00311(13) & -0.00180(12) \\ \mathrm{I} 2 & 0.0231(2) & 0.0219(2) & 0.0252(2) & -0.00092(15) & -0.00093(16) & -0.00113(16) \\ \mathrm{B} 2 & 0.0266(3) & 0.0199(3) & 0.0267(3) & -0.0050(2) & 0.0032(3) & -0.0015(2) \\ \mathrm{N} 1^{\prime} & 0.028(3) & 0.014(2) & 0.017(2) & -0.001(2) & 0.001(2) & 0.0007(19) \\ \mathrm{N} 2^{\prime} & 0.028(3) & 0.015(2) & 0.022(3) & 0.001(2) & -0.001(2) & -0.002(2) \\ \mathrm{C} 1^{\prime} & 0.031(3) & 0.014(3) & 0.015(3) & 0.004(2) & 0.000(2) & 0.000(2) \\ \mathrm{C} 2^{\prime} & 0.024(3) & 0.020(3) & 0.022(3) & 0.000(2) & 0.005(2) & 0.000(2) \\ \mathrm{C} 3^{\prime} & 0.025(3) & 0.017(3) & 0.021(3) & 0.000(2) & -0.002(2) & -0.002(2) \\ \mathrm{C} 4^{\prime} & 0.026(3) & 0.016(3) & 0.026(3) & 0.007(2) & 0.008(3) & 0.002(2) \\ \mathrm{C}^{\prime} & 0.031(4) & 0.019(3) & 0.039(4) & 0.008(3) & 0.010(3) & 0.001(3) \\ \mathrm{C}^{\prime} & 0.031(4) & 0.015(3) & 0.029(3) & 0.002(3) & -0.005(3) & -0.001(3) \\ \mathrm{C}^{\prime} & 0.025(3) & 0.022(3) & 0.038(4) & 0.000(3) & 0.008(3) & 0.008(3) \\ & & & & & & \end{array}$

Geometric parameters $\left(\AA,{ }^{o}\right)$

\begin{tabular}{llll}
\hline $\mathrm{Au} 1-\mathrm{C} 1^{\mathrm{i}}$ & $2.033(7)$ & $\mathrm{N} 2^{\prime}-\mathrm{C} 6^{\prime}$ & $1.459(9)$ \\
$\mathrm{Au} 1-\mathrm{C} 1$ & $2.033(7)$ & $\mathrm{C} 2^{\prime}-\mathrm{C} 3^{\prime}$ & $1.353(9)$ \\
$\mathrm{Au} 1-\mathrm{I} 1^{\mathrm{i}}$ & $2.6564(5)$ & $\mathrm{C} 4^{\prime}-\mathrm{C} 5^{\prime}$ & $1.519(10)$ \\
$\mathrm{Au} 1-\mathrm{I} 1$ & $2.6564(5)$ & $\mathrm{C} 6^{\prime}-\mathrm{C}^{\prime}$ & $1.519(11)$ \\
$\mathrm{I} 1-\mathrm{I} 2^{\mathrm{ii}}$ & $3.5136(7)$ & $\mathrm{C} 3-\mathrm{H} 3$ & 0.9500 \\
$\mathrm{Br} 1-\mathrm{C} 2$ & $1.870(7)$ & $\mathrm{C} 4-\mathrm{H} 4 \mathrm{~A}$ & 0.9900 \\
$\mathrm{Br} 1-\mathrm{I} 2$ & $3.4347(8)$ & $\mathrm{C} 4-\mathrm{H} 4 \mathrm{~B}$ & 0.9900 \\
$\mathrm{~N} 1-\mathrm{C} 1$ & $1.347(8)$ & $\mathrm{C} 5-\mathrm{H} 5 \mathrm{~A}$ & 0.9800 \\
$\mathrm{~N} 1-\mathrm{C} 2$ & $1.397(9)$ & $\mathrm{C} 5-\mathrm{H} 5 \mathrm{~B}$ & 0.9800 \\
$\mathrm{~N} 1-\mathrm{C} 4$ & $1.464(10)$ & $\mathrm{C} 5-\mathrm{H} 5 \mathrm{C}$ & 0.9800 \\
$\mathrm{~N} 2-\mathrm{C} 1$ & $1.351(9)$ & $\mathrm{C} 6-\mathrm{H} 6 \mathrm{~A}$ & 0.9900 \\
$\mathrm{~N} 2-\mathrm{C} 3$ & $1.385(8)$ & $\mathrm{C} 6-\mathrm{H} 6 \mathrm{~B}$ & 0.9900 \\
$\mathrm{~N} 2-\mathrm{C} 6$ & $1.479(9)$ & $\mathrm{C} 7-\mathrm{H} 7 \mathrm{~A}$ & 0.9800 \\
$\mathrm{C} 2-\mathrm{C} 3$ & $1.348(10)$ & $\mathrm{C} 7-\mathrm{H} 7 \mathrm{~B}$ & 0.9800 \\
$\mathrm{C} 4-\mathrm{C} 5$ & $1.530(12)$ & $\mathrm{C} 7-\mathrm{H} 7 \mathrm{C}$ & 0.9800 \\
$\mathrm{C} 6-\mathrm{C} 7$ & $1.504(11)$ & $\mathrm{C} 3^{\prime}-\mathrm{H} 3^{\prime}$ & 0.9500 \\
$\mathrm{Au} 2-\mathrm{C} 1^{\prime}$ & $2.018(7)$ & $\mathrm{C} 4^{\prime}-\mathrm{H} 4^{\prime} 1$ & 0.9900 \\
$\mathrm{Au} 2-\mathrm{C} 1^{\prime \text { iii }}$ & $2.018(7)$ & $\mathrm{C} 4^{\prime}-\mathrm{H} 4^{\prime 2}$ & 0.9900 \\
$\mathrm{I} 2-\mathrm{Br} 2$ & $3.5575(8)$ & $\mathrm{C} 5^{\prime}-\mathrm{H} 5^{\prime} 1$ & 0.9800 \\
$\mathrm{I} 2-\mathrm{Au} 1^{\mathrm{ii}}$ & $4.1539(5)$ & $\mathrm{C} 5^{\prime}-\mathrm{H} 5^{\prime 2}$ & 0.9800 \\
$\mathrm{Br} 2-\mathrm{C} 2^{\prime}$ & $1.871(7)$ & $\mathrm{C} 5^{\prime}-\mathrm{H} 5^{\prime} 3$ & 0.9800 \\
$\mathrm{~N} 1^{\prime}-\mathrm{C} 1^{\prime}$ & $1.360(8)$ & $\mathrm{C} 6^{\prime}-\mathrm{H} 6^{\prime} 1$ & 0.9900 \\
$\mathrm{~N} 1^{\prime}-\mathrm{C} 2^{\prime}$ & $1.383(9)$ & $\mathrm{C} 6^{\prime}-\mathrm{H} 6^{\prime 2}$ & 0.9900 \\
$\mathrm{~N} 1^{\prime}-\mathrm{C} 4^{\prime}$ & $1.469(8)$ & $\mathrm{C} 7^{\prime}-\mathrm{H} 7^{\prime} 1$ & 0.9800 \\
$\mathrm{~N} 2^{\prime}-\mathrm{C} 1^{\prime}$ & $1.354(9)$ & $\mathrm{C} 7^{\prime}-\mathrm{H} 7^{\prime 2}$ & 0.9800 \\
$\mathrm{~N} 2^{\prime}-\mathrm{C} 3^{\prime}$ & $1.386(9)$ & $\mathrm{C} 7^{\prime}-\mathrm{H} 7^{\prime} 3$ & 0.9800 \\
& & &
\end{tabular}




\begin{tabular}{|c|c|c|c|}
\hline $\mathrm{C} 1-\mathrm{Au} 1-\mathrm{C} 1$ & 180.0 & $\mathrm{~N} 2-\mathrm{C} 3-\mathrm{H} 3$ & 126.7 \\
\hline $\mathrm{C} 1^{\mathrm{i}}-\mathrm{Au} 1-\mathrm{I} 1^{\mathrm{i}}$ & 90.95 (18) & $\mathrm{N} 1-\mathrm{C} 4-\mathrm{H} 4 \mathrm{~A}$ & 109.1 \\
\hline $\mathrm{C} 1-\mathrm{Au} 1-\mathrm{I} 1^{\mathrm{i}}$ & 89.05 (18) & $\mathrm{C} 5-\mathrm{C} 4-\mathrm{H} 4 \mathrm{~A}$ & 109.1 \\
\hline $\mathrm{C} 1$ - $\mathrm{Au} 1-\mathrm{I} 1$ & 89.05 (18) & $\mathrm{N} 1-\mathrm{C} 4-\mathrm{H} 4 \mathrm{~B}$ & 109.1 \\
\hline $\mathrm{C} 1-\mathrm{Au} 1-\mathrm{I} 1$ & 90.95 (18) & $\mathrm{C} 5-\mathrm{C} 4-\mathrm{H} 4 \mathrm{~B}$ & 109.1 \\
\hline $\mathrm{I} 1{ }^{\mathrm{i}}-\mathrm{Au} 1-\mathrm{I} 1$ & 180.0 & $\mathrm{H} 4 \mathrm{~A}-\mathrm{C} 4-\mathrm{H} 4 \mathrm{~B}$ & 107.8 \\
\hline $\mathrm{Au} 1-\mathrm{I} 1-\mathrm{I} 2^{\mathrm{ii}}$ & $176.29(2)$ & $\mathrm{C} 4-\mathrm{C} 5-\mathrm{H} 5 \mathrm{~A}$ & 109.5 \\
\hline $\mathrm{C} 2-\mathrm{Br} 1-\mathrm{I} 2$ & $179.5(2)$ & $\mathrm{C} 4-\mathrm{C} 5-\mathrm{H} 5 \mathrm{~B}$ & 109.5 \\
\hline $\mathrm{C} 1-\mathrm{N} 1-\mathrm{C} 2$ & $109.5(6)$ & $\mathrm{H} 5 \mathrm{~A}-\mathrm{C} 5-\mathrm{H} 5 \mathrm{~B}$ & 109.5 \\
\hline $\mathrm{C} 1-\mathrm{N} 1-\mathrm{C} 4$ & $124.8(6)$ & $\mathrm{C} 4-\mathrm{C} 5-\mathrm{H} 5 \mathrm{C}$ & 109.5 \\
\hline $\mathrm{C} 2-\mathrm{N} 1-\mathrm{C} 4$ & $125.3(6)$ & $\mathrm{H} 5 \mathrm{~A}-\mathrm{C} 5-\mathrm{H} 5 \mathrm{C}$ & 109.5 \\
\hline $\mathrm{C} 1-\mathrm{N} 2-\mathrm{C} 3$ & $110.4(6)$ & $\mathrm{H} 5 \mathrm{~B}-\mathrm{C} 5-\mathrm{H} 5 \mathrm{C}$ & 109.5 \\
\hline $\mathrm{C} 1-\mathrm{N} 2-\mathrm{C} 6$ & $125.6(6)$ & $\mathrm{N} 2-\mathrm{C} 6-\mathrm{H} 6 \mathrm{~A}$ & 109.3 \\
\hline $\mathrm{C} 3-\mathrm{N} 2-\mathrm{C} 6$ & $124.0(6)$ & $\mathrm{C} 7-\mathrm{C} 6-\mathrm{H} 6 \mathrm{~A}$ & 109.3 \\
\hline $\mathrm{N} 1-\mathrm{C} 1-\mathrm{N} 2$ & $106.2(6)$ & $\mathrm{N} 2-\mathrm{C} 6-\mathrm{H} 6 \mathrm{~B}$ & 109.3 \\
\hline $\mathrm{N} 1-\mathrm{C} 1-\mathrm{Au} 1$ & $126.4(5)$ & $\mathrm{C} 7-\mathrm{C} 6-\mathrm{H} 6 \mathrm{~B}$ & 109.3 \\
\hline $\mathrm{N} 2-\mathrm{C} 1-\mathrm{Au} 1$ & $127.4(5)$ & $\mathrm{H} 6 \mathrm{~A}-\mathrm{C} 6-\mathrm{H} 6 \mathrm{~B}$ & 107.9 \\
\hline $\mathrm{C} 3-\mathrm{C} 2-\mathrm{N} 1$ & $107.2(6)$ & $\mathrm{C} 6-\mathrm{C} 7-\mathrm{H} 7 \mathrm{~A}$ & 109.5 \\
\hline $\mathrm{C} 3-\mathrm{C} 2-\mathrm{Br} 1$ & $129.9(6)$ & $\mathrm{C} 6-\mathrm{C} 7-\mathrm{H} 7 \mathrm{~B}$ & 109.5 \\
\hline $\mathrm{N} 1-\mathrm{C} 2-\mathrm{Br} 1$ & $122.9(5)$ & $\mathrm{H} 7 \mathrm{~A}-\mathrm{C} 7-\mathrm{H} 7 \mathrm{~B}$ & 109.5 \\
\hline $\mathrm{C} 2-\mathrm{C} 3-\mathrm{N} 2$ & $106.6(6)$ & $\mathrm{C} 6-\mathrm{C} 7-\mathrm{H} 7 \mathrm{C}$ & 109.5 \\
\hline $\mathrm{N} 1-\mathrm{C} 4-\mathrm{C} 5$ & $112.6(7)$ & $\mathrm{H} 7 \mathrm{~A}-\mathrm{C} 7-\mathrm{H} 7 \mathrm{C}$ & 109.5 \\
\hline $\mathrm{N} 2-\mathrm{C} 6-\mathrm{C} 7$ & $111.8(6)$ & $\mathrm{H} 7 \mathrm{~B}-\mathrm{C} 7-\mathrm{H} 7 \mathrm{C}$ & 109.5 \\
\hline $\mathrm{C} 1^{\prime}-\mathrm{Au} 2-\mathrm{C}^{\prime}{ }^{\prime \mathrm{iii}}$ & 180.0 & $\mathrm{C} 2^{\prime}-\mathrm{C} 3^{\prime}-\mathrm{H} 3^{\prime}$ & 127.0 \\
\hline $\mathrm{Br} 1-\mathrm{I} 2-\mathrm{I} 1^{\mathrm{ii}}$ & $97.240(19)$ & $\mathrm{N} 2^{\prime}-\mathrm{C}^{\prime}-\mathrm{H} 3^{\prime}$ & 127.0 \\
\hline $\mathrm{Br} 1-\mathrm{I} 2-\mathrm{Br} 2$ & $169.87(2)$ & $\mathrm{N} 1^{\prime}-\mathrm{C} 4^{\prime}-\mathrm{H} 4^{\prime} 1$ & 109.3 \\
\hline $\mathrm{I} 1{ }^{\mathrm{ii}}-\mathrm{I} 2-\mathrm{Br} 2$ & $72.852(17)$ & $\mathrm{C} 5^{\prime}-\mathrm{C} 4^{\prime}-\mathrm{H} 4^{\prime} 1$ & 109.3 \\
\hline $\mathrm{Br} 1-\mathrm{I} 2-\mathrm{Au} 2^{\mathrm{iv}}$ & 74.604 (16) & $\mathrm{N} 1^{\prime}-\mathrm{C} 4^{\prime}-\mathrm{H} 4^{\prime} 2$ & 109.3 \\
\hline $\mathrm{I} 1^{\mathrm{ii}}-\mathrm{I} 2-\mathrm{Au} 2^{\mathrm{iv}}$ & $168.488(16)$ & $\mathrm{C} 5^{\prime}-\mathrm{C} 4^{\prime}-\mathrm{H} 4^{\prime} 2$ & 109.3 \\
\hline $\mathrm{Br} 2-\mathrm{I} 2-\mathrm{Au} 2^{\mathrm{iv}}$ & $114.871(16)$ & $\mathrm{H} 4^{\prime} 1-\mathrm{C} 4^{\prime}-\mathrm{H}^{\prime}{ }^{\prime} 2$ & 108.0 \\
\hline $\mathrm{C} 2^{\prime}-\mathrm{Br} 2-\mathrm{I} 2$ & $177.0(2)$ & $\mathrm{C} 4^{\prime}-\mathrm{C} 5^{\prime}-\mathrm{H} 5^{\prime} 1$ & 109.5 \\
\hline $\mathrm{C} 1^{\prime}-\mathrm{N} 1^{\prime}-\mathrm{C} 2^{\prime}$ & $110.4(6)$ & $\mathrm{C} 4^{\prime}-\mathrm{C} 5^{\prime}-\mathrm{H} 5^{\prime} 2$ & 109.5 \\
\hline $\mathrm{C} 1^{\prime}-\mathrm{N} 1^{\prime}-\mathrm{C} 4^{\prime}$ & $123.3(6)$ & $\mathrm{H} 5^{\prime} 1-\mathrm{C} 5^{\prime}-\mathrm{H} 5^{\prime} 2$ & 109.5 \\
\hline $\mathrm{C} 2^{\prime}-\mathrm{N} 1^{\prime}-\mathrm{C} 4^{\prime}$ & $126.1(6)$ & $\mathrm{C} 4^{\prime}-\mathrm{C} 5^{\prime}-\mathrm{H} 5^{\prime} 3$ & 109.5 \\
\hline $\mathrm{C} 1^{\prime}-\mathrm{N} 2^{\prime}-\mathrm{C} 3^{\prime}$ & $111.4(6)$ & $\mathrm{H} 5^{\prime} 1-\mathrm{C} 5^{\prime}-\mathrm{H} 5^{\prime} 3$ & 109.5 \\
\hline $\mathrm{C} 1^{\prime}-\mathrm{N} 2^{\prime}-\mathrm{C} 6^{\prime}$ & $124.9(6)$ & $\mathrm{H} 5^{\prime} 2-\mathrm{C}^{\prime}-\mathrm{H} 5^{\prime} 3$ & 109.5 \\
\hline $\mathrm{C} 3^{\prime}-\mathrm{N} 2^{\prime}-\mathrm{C} 6^{\prime}$ & $123.3(6)$ & $\mathrm{N} 2^{\prime}-\mathrm{C} 6^{\prime}-\mathrm{H} 6^{\prime} 1$ & 109.4 \\
\hline $\mathrm{N} 2^{\prime}-\mathrm{C} 1^{\prime}-\mathrm{N} 1^{\prime}$ & $104.7(6)$ & $\mathrm{C} 7^{\prime}-\mathrm{C} 6^{\prime}-\mathrm{H} 6^{\prime} 1$ & 109.4 \\
\hline $\mathrm{N} 2^{\prime}-\mathrm{C} 1^{\prime}-\mathrm{Au} 2$ & $128.9(5)$ & $\mathrm{N} 2^{\prime}-\mathrm{C} 6^{\prime}-\mathrm{H} 6^{\prime} 2$ & 109.4 \\
\hline $\mathrm{N} 1^{\prime}-\mathrm{C} 1^{\prime}-\mathrm{Au} 2$ & $126.4(5)$ & $\mathrm{C} 7^{\prime}-\mathrm{C} 6^{\prime}-\mathrm{H} 6^{\prime} 2$ & 109.4 \\
\hline $\mathrm{C} 3^{\prime}-\mathrm{C} 2^{\prime}-\mathrm{N} 1^{\prime}$ & $107.4(6)$ & $\mathrm{H} 6^{\prime} 1-\mathrm{C} 6^{\prime}-\mathrm{H} 6^{\prime} 2$ & 108.0 \\
\hline $\mathrm{C} 3^{\prime}-\mathrm{C} 2^{\prime}-\mathrm{Br} 2$ & $128.7(5)$ & $\mathrm{C} 6^{\prime}-\mathrm{C} 7^{\prime}-\mathrm{H} 7^{\prime} 1$ & 109.5 \\
\hline $\mathrm{N} 1^{\prime}-\mathrm{C} 2^{\prime}-\mathrm{Br} 2$ & $123.9(5)$ & $\mathrm{C} 6{ }^{\prime}-\mathrm{C}^{\prime}-\mathrm{H} 7^{\prime} 2$ & 109.5 \\
\hline $\mathrm{C} 2^{\prime}-\mathrm{C} 3^{\prime}-\mathrm{N} 2^{\prime}$ & $105.9(6)$ & $\mathrm{H} 7^{\prime} 1-\mathrm{C}^{\prime}-\mathrm{H} 7^{\prime} 2$ & 109.5 \\
\hline $\mathrm{N} 1^{\prime}-\mathrm{C} 4^{\prime}-\mathrm{C} 5^{\prime}$ & $111.4(6)$ & $\mathrm{C} 6^{\prime}-\mathrm{C} 7^{\prime}-\mathrm{H} 7^{\prime} 3$ & 109.5 \\
\hline $\mathrm{N} 2^{\prime}-\mathrm{C} 6^{\prime}-\mathrm{C} 7^{\prime}$ & $111.0(6)$ & $\mathrm{H} 7^{\prime} 1-\mathrm{C}^{\prime}-\mathrm{H}^{\prime} 3$ & 109.5 \\
\hline $\mathrm{C} 2-\mathrm{C} 3-\mathrm{H} 3$ & 126.7 & $\mathrm{H} 77^{\prime} 2-\mathrm{C}^{\prime}-\mathrm{H}^{\prime} 3$ & 109.5 \\
\hline
\end{tabular}




$\begin{array}{llll}\mathrm{C} 2-\mathrm{N} 1-\mathrm{C} 1-\mathrm{N} 2 & -0.4(7) & \mathrm{C} 3^{\prime}-\mathrm{N} 2^{\prime}-\mathrm{C} 1^{\prime}-\mathrm{N} 1^{\prime} & -0.4(8) \\ \mathrm{C} 4-\mathrm{N} 1-\mathrm{C} 1-\mathrm{N} 2 & -174.5(6) & \mathrm{C} 6^{\prime}-\mathrm{N} 2^{\prime}-\mathrm{C} 1^{\prime}-\mathrm{N} 1^{\prime} & -173.8(6) \\ \mathrm{C} 2-\mathrm{N} 1-\mathrm{C} 1-\mathrm{Au} 1 & -179.8(5) & \mathrm{C} 3^{\prime}-\mathrm{N} 2^{\prime}-\mathrm{C} 1^{\prime}-\mathrm{Au} 2 & 178.6(5) \\ \mathrm{C} 4-\mathrm{N} 1-\mathrm{C} 1-\mathrm{Au} 1 & 6.2(10) & \mathrm{C} 6^{\prime}-\mathrm{N} 2^{\prime}-\mathrm{C} 1^{\prime}-\mathrm{Au} 2 & 5.3(10) \\ \mathrm{C} 3-\mathrm{N} 2-\mathrm{C} 1-\mathrm{N} 1 & 1.0(8) & \mathrm{C} 2^{\prime}-\mathrm{N} 1^{\prime}-\mathrm{C} 1^{\prime}-\mathrm{N} 2^{\prime} & 0.4(7) \\ \mathrm{C} 6-\mathrm{N} 2-\mathrm{C} 1-\mathrm{N} 1 & -179.5(6) & \mathrm{C} 4^{\prime}-\mathrm{N} 1^{\prime}-\mathrm{C} 1^{\prime}-\mathrm{N} 2^{\prime} & 176.4(6) \\ \mathrm{C} 3-\mathrm{N} 2-\mathrm{C} 1-\mathrm{Au} 1 & -179.7(5) & \mathrm{C} 2^{\prime}-\mathrm{N} 1^{\prime}-\mathrm{C} 1^{\prime}-\mathrm{Au} 2 & -178.8(5) \\ \mathrm{C} 6-\mathrm{N} 2-\mathrm{C} 1-\mathrm{Au} 1 & -0.2(10) & \mathrm{C} 4^{\prime}-\mathrm{N} 1^{\prime}-\mathrm{C} 1^{\prime}-\mathrm{Au} 2 & -2.7(9) \\ \mathrm{C} 1-\mathrm{N} 1-\mathrm{C} 2-\mathrm{C} 3 & -0.3(8) & \mathrm{C} 1^{\prime}-\mathrm{N} 1^{\prime}-\mathrm{C} 2^{\prime}-\mathrm{C} 3^{\prime} & -0.1(8) \\ \mathrm{C} 4-\mathrm{N} 1-\mathrm{C} 2-\mathrm{C} 3 & 173.8(7) & \mathrm{C} 4^{\prime}-\mathrm{N} 1^{\prime}-\mathrm{C} 2^{\prime}-\mathrm{C} 3^{\prime} & -176.1(6) \\ \mathrm{C} 1-\mathrm{N} 1-\mathrm{C} 2-\mathrm{Br} 1 & 179.1(5) & \mathrm{C} 1^{\prime}-\mathrm{N} 1^{\prime}-\mathrm{C} 2^{\prime}-\mathrm{Br} 2 & 178.1(5) \\ \mathrm{C} 4-\mathrm{N} 1-\mathrm{C} 2-\mathrm{Br} 1 & -6.8(10) & \mathrm{C} 4^{\prime}-\mathrm{N} 1^{\prime}-\mathrm{C} 2^{\prime}-\mathrm{Br} 2 & 2.2(9) \\ \mathrm{N} 1-\mathrm{C} 2-\mathrm{C} 3-\mathrm{N} 2 & 0.9(8) & \mathrm{N} 1^{\prime}-\mathrm{C} 2^{\prime}-\mathrm{C} 3^{\prime}-\mathrm{N} 2^{\prime} & -0.1(8) \\ \mathrm{B} 1-\mathrm{C} 2-\mathrm{C} 3-\mathrm{N} 2 & -178.5(5) & \mathrm{B} 2-\mathrm{C} 2^{\prime}-\mathrm{C} 3^{\prime}-\mathrm{N} 2^{\prime} & -178.3(5) \\ \mathrm{C} 1-\mathrm{N} 2-\mathrm{C} 3-\mathrm{C} 2 & -1.2(8) & \mathrm{C} 1^{\prime}-\mathrm{N} 2^{\prime}-\mathrm{C} 3^{\prime}-\mathrm{C} 2^{\prime} & 0.4(8) \\ \mathrm{C} 6-\mathrm{N} 2-\mathrm{C} 3-\mathrm{C} 2 & 179.3(6) & \mathrm{C} 6^{\prime}-\mathrm{N} 2^{\prime}-\mathrm{C} 3^{\prime}-\mathrm{C} 2^{\prime} & 173.8(6) \\ \mathrm{C} 1-\mathrm{N} 1-\mathrm{C} 4-\mathrm{C} 5 & 89.5(8) & \mathrm{C} 1^{\prime}-\mathrm{N} 1^{\prime}-\mathrm{C} 4^{\prime}-\mathrm{C} 5^{\prime} & -79.6(8) \\ \mathrm{C} 2-\mathrm{N} 1-\mathrm{C} 4-\mathrm{C} 5 & -83.7(9) & \mathrm{C} 2^{\prime}-\mathrm{N} 1^{\prime}-\mathrm{C} 4^{\prime}-\mathrm{C} 5^{\prime} & 95.8(8) \\ \mathrm{C} 1-\mathrm{N} 2-\mathrm{C} 6-\mathrm{C} 7 & 76.1(9) & \mathrm{C} 1^{\prime}-\mathrm{N} 2^{\prime}-\mathrm{C} 6^{\prime}-\mathrm{C} 7^{\prime} & 97.4(8) \\ \mathrm{C} 3-\mathrm{N} 2-\mathrm{C} 6-\mathrm{C} 7 & -104.5(8) & \mathrm{C} 3^{\prime}-\mathrm{N} 2^{\prime}-\mathrm{C} 6^{\prime}-\mathrm{C} 7^{\prime} & -75.2(9)\end{array}$

Symmetry codes: (i) $-x-1,-y-1,-z$; (ii) $-x,-y,-z$; (iii) $-x+2,-y+2,-z+1$; (iv) $x-1, y-1, z$.

Hydrogen-bond geometry $\left(A,{ }^{\circ}\right)$

\begin{tabular}{lllll}
\hline$D-\mathrm{H} \cdots A$ & $D-\mathrm{H}$ & $\mathrm{H} \cdots A$ & $D \cdots A$ & $D-\mathrm{H} \cdots A$ \\
\hline $\mathrm{C} 3-\mathrm{H} 3 \cdots \mathrm{I} 1^{\mathrm{v}}$ & 0.95 & 3.28 & $3.913(7)$ & 126 \\
$\mathrm{C} 3-\mathrm{H} 3 \cdots \mathrm{I} 2^{\text {vi }}$ & 0.95 & 3.22 & $4.011(7)$ & 142 \\
$\mathrm{C} 4-\mathrm{H} 4 A \cdots \mathrm{I} 1$ & 0.99 & 3.28 & $4.002(7)$ & 132 \\
$\mathrm{C} 6-\mathrm{H} 6 A \cdots \mathrm{I} 1^{\mathrm{i}}$ & 0.99 & 3.16 & $3.925(7)$ & 136 \\
$\mathrm{C} 6-\mathrm{H} 6 B^{\cdots} \cdots 2^{\text {vi }}$ & 0.99 & 3.11 & $4.064(7)$ & 163 \\
$\mathrm{C} 7-\mathrm{H} 7 C^{\prime} \cdots \mathrm{I} 1^{\mathrm{v}}$ & 0.98 & 3.29 & $4.051(9)$ & 136 \\
$\mathrm{C} 3^{\prime}-\mathrm{H} 3^{\prime} \cdots \mathrm{I} 2^{\text {vii }}$ & 0.95 & 3.08 & $3.916(7)$ & 148 \\
$\mathrm{C} 4^{\prime}-\mathrm{H} 4^{\prime} 2 \cdots \mathrm{I} 2^{\text {viii }}$ & 0.99 & 3.10 & $3.883(7)$ & 137 \\
$\mathrm{C}^{\prime}-\mathrm{H} 7^{\prime} 1 \cdots \mathrm{I} 2^{\text {ix }}$ & 0.98 & 3.19 & $4.039(7)$ & 146
\end{tabular}

Symmetry codes: (i) $-x-1,-y-1,-z$; (v) $-x,-y-1,-z$; (vi) $x, y-1, z$; (vii) $x, y+1, z$; (viii) $-x+1,-y+1,-z+1$; (ix) $x+1, y+1, z$. 